\title{
An efficient central DOA tracking algorithm for multiple incoherently distributed sources
}

\author{
Sonia Ben Hassen ${ }^{1 *}$ and Abdelaziz Samet ${ }^{1,2}$
}

\begin{abstract}
In this paper, we develop a new tracking method for the direction of arrival (DOA) parameters assuming multiple incoherently distributed (ID) sources. The new approach is based on a simple covariance fitting optimization technique exploiting the central and noncentral moments of the source angular power densities to estimate the central DOAs. The current estimates are treated as measurements provided to the Kalman filter that model the dynamic property of directional changes for the moving sources. Then, the covariance-fitting-based algorithm and the Kalman filtering theory are combined to formulate an adaptive tracking algorithm. Our algorithm is compared to the fast approximated power iteration-total least square-estimation of signal parameters via rotational invariance technique (FAPI-TLS-ESPRIT) algorithm using the TLS-ESPRIT method and the subspace updating via FAPI-algorithm. It will be shown that the proposed algorithm offers an excellent DOA tracking performance and outperforms the FAPI-TLS-ESPRIT method especially at low signal-to-noise ratio (SNR) values. Moreover, the performances of the two methods increase as the SNR values increase. This increase is more prominent with the FAPI-TLS-ESPRIT method. However, their performances degrade when the number of sources increases. It will be also proved that our method depends on the form of the angular distribution function when tracking the central DOAs. Finally, it will be shown that the more the sources are spaced, the more the proposed method can exactly track the DOAs.
\end{abstract}

Keywords: Tracking DOAs, Incoherently distributed sources, Covariance fitting technique, Kalman filter

\section{Review}

\subsection{Introduction}

The most commonly considered system model in the direction of arrival (DOA)-finding techniques is the point source model where the signals are assumed to be generated from far-field point sources [1-4]. However, in real surroundings, especially in modern wireless communication systems, local scattering in the source vicinity causes angular spreading. Therefore, the researchers considered a more realistic signal model called spatially distributed source model. Depending on the nature of scattering, distributed sources have been classified into two types: coherently and incoherently distributed (CD and ID) sources $[5,6]$. For ID source model contrary to the CD case, the rank of the noise-free covariance matrix is different to the number of sources. Moreover, it increases with the angular spread. Therefore, traditional subspace-based methods become not applicable in this case. To deal with

\footnotetext{
*Correspondence: sonia.benhassen@yahoo.fr

1 Tunisia Polytechnic School, B.P. 743-2078, La Marsa, Tunisia

Full list of author information is available at the end of the article
}

this problem, efforts have been directed to specifically design new techniques to estimate the angular parameters for ID sources. Some of them that are able to handle a single ID source were developed in [7-13]. Many other estimators were also developed to estimate the angular parameters of multiple ID sources. In fact, some subspace methods were proposed in $[5,14,15]$ wherein the effective dimension of the signal subspace is defined as the number of the first eigenvalues of the noise-free covariance matrix where most of the signal energy is concentrated.

Despite their high accuracy, these methods suffer from a heavy computational load. To reduce the complexity, the so-called TLS-ESPRIT algorithm was derived in [16] which is based on the total least square (TLS) estimation of signal parameters via rotational invariance technique (ESPRIT).

Moreover, more computationally attractive but less efficient methods using the beamforming techniques were developed in $[17,18]$. Later, Shahbazpanahi et al. proposed a new algorithm based on central and noncentral moments of the sources angular power densities [19]. In

\section{焦 Springer}

(c) 2015 Hassen and Samet. Open Access This article is distributed under the terms of the Creative Commons Attribution 4.0 International License (http://creativecommons.org/licenses/by/4.0/), which permits unrestricted use, distribution, and reproduction in any medium, provided you give appropriate credit to the original author(s) and the source, provide a link to the Creative Commons license, and indicate if changes were made. 
addition to its reduced complexity, this method is applicable to the multisource scenarios with different angular power densities. Recently, authors derived in [20] a new algorithm for the estimation of the angular parameters of multiple ID sources. It exploits the property of the inverse of the covariance matrix to estimate the angular parameters.

Despite its efficiency, this method requires that the multiple sources have an identical angular distribution. Moreover, it estimates the angular parameters (central DOAs and angular spreads) together with a 2D search.

All the aforementioned techniques assume the sources to maintain the same positions, and then their central DOAs are constant in time. This assumption is, however, too restrictive in wireless communication applications where mobile terminals often change their spatial positions. Therefore, several approaches have been proposed to deal with the problem of tracking the discrete DOAs at different time points. Most of them are based on subspace tracking techniques such as the projection approximate subspace tracking (PAST) of [21] and the orthonormal projection approximate subspace tracking (OPAST) of [22].

In this context, authors have proposed in [23] a simple DOA tracking scheme involving the PAST-algorithm and the Kalman filter to track the DOAs. Later, in [24], a fast implementation of the power iterations method for subspace tracking (FAPI) was derived. Recently, the researchers proposed in [25-27] new tracking algorithms based on some modifications of the Kalman filter and the existing subspace tracking techniques. All these tracking algorithms are limited to the point source model.

To deal with the problem of estimating the time-varying DOAs in scattering channels, a simple DOA tracking method based on the TLS-ESPRIT [16] and subspace updating via FAPI algorithm has been recently proposed in [28] for ID sources. Despite its relatively reduced computational cost, this method was shown to exhibit poor tracking success rate at low signal-to-noise ratios (SNRs). More recently, a DOA tracking method based on a support vector regression approach was developed in [29] for coherently distributed source. Therefore, we aim in this paper to consider the problem of tracking DOAs at different time points in scattering channels. As the assumption of uncorrelated ID sources has been shown to be relevant in wireless communications environments with a high base station than the CD source case [30], we assume in this paper that the sources are ID.

We then derive a new method that outperforms the method derived in [28]. This method is based on a simple covariance fitting optimization technique as developed in [19] to estimate the central DOAs in each observed time interval. In fact, this technique can estimate the central DOAs regardless of the angular spreads, and it is applicable to the multisource scenarios with different angular power densities. The new tracking method also uses the famous Kalman filter to model the mobility of the sources and track the different DOAs during the tracking period. The Kalman filter (KF) can reduce estimation errors and avoid the data association problem when applied to angle tracking due to the nature of prediction correction.

This paper is organized as follows. In subsection 1.2, we introduce the system model that will be used throughout the article and we define the problem in terms of notation and assumptions. In subsection 1.3, we formulate the new algorithm. In subsection 1.4, the proposed algorithm is compared to the one of [28] through computer simulations.

Throughout this paper, matrices and vectors are represented by bold upper and lowercase characters, respectively. Vectors are, by default, in column orientation, while $(.)^{*},(.)^{T}$ and $(.)^{H}$ refer to conjugate, transpose, and conjugate transpose, respectively. Moreover, $E\{$.$\} and \operatorname{tr}($. stand for the statistical expectation and trace operators, respectively. Furthermore, eig(.) and $\operatorname{diag}($.$) represent the$ eigenvalues of a matrix and the diagonal matrix of a vector, respectively. Finally, $\otimes$ stand for the Kronecker operator.

\subsection{System model}

Consider a uniform linear array of $L$ identical sensors (i.e., with the same gain, phase, and sensitivity pattern). The array receives signals distributed from $K$ ID narrowband ${ }^{1}$ far-field sources with the same central frequency $\omega_{0}$. Then, the output of the $l$ th array sensor can be modelled as a complex signal as follows [16-20]:

$$
x_{l}(t)=\sum_{k=1}^{K} \int_{-\pi / 2}^{\pi / 2} a_{l}(\theta) s_{k}\left(\theta, \boldsymbol{\psi}_{k}, t\right) d \theta+n_{l}(t),
$$

where $a_{l}(\theta)$ is the response of the $l$ th sensor to a unit energy source emitting from the direction $\theta$. Moreover, $s_{k}\left(\theta, \boldsymbol{\psi}_{k}, t\right)$ is the angular signal distribution of the $k$ th source that is parameterized by the parameter vector, $\psi_{k}=\left[\bar{\theta}_{k}, \sigma_{k}\right]^{T}$, containing its central DOA, $\bar{\theta}_{k}$, and angular spread, $\sigma_{k}$. For each $k$ th source, the central DOA is defined as the mass center of the corresponding normalized angular power density, $\rho_{k}($.$) , as follows:$

$$
\bar{\theta}_{k}=\int_{-\frac{\pi}{2}}^{\frac{\pi}{2}} \theta \rho_{k}\left(\theta, \boldsymbol{\psi}_{k}\right) d \theta,
$$

where $\rho_{k}\left(\theta, \boldsymbol{\psi}_{k}\right)$ satisfies the following identity:

$$
\int_{-\pi / 2}^{\pi / 2} \rho_{k}\left(\theta, \psi_{k}\right) d \theta=1, \quad k=1,2, \ldots, K
$$


Furthermore, the angular spread for each $k$ th source is obtained from the second central moment as follows:

$$
\sigma_{k}=\left(\int_{-\pi / 2}^{\pi / 2}\left(\theta-\bar{\theta}_{k}\right)^{2} \rho_{k}\left(\theta, \boldsymbol{\psi}_{k}\right) d \theta\right)^{1 / 2} .
$$

Finally, $n_{l}(t)$ represents the additive zero-mean, Gaussian distributed, circular, spatially and temporally white noise (i.e., uncorrelated between the receiving antenna elements and between different snapshots).

Stacking the received data over the $L$ sensors, at given time instant $t$, in a vector $\boldsymbol{x}(t)=\left[x_{1}(t), x_{2}(t), \ldots, x_{L}(t)\right]^{T}$, it follows from (1) that:

$$
\boldsymbol{x}(t)=\sum_{k=1}^{K} \int_{-\pi / 2}^{\pi / 2} \boldsymbol{a}(\theta) s_{k}\left(\theta, \boldsymbol{\psi}_{k}, t\right) d \theta+\boldsymbol{n}(t),
$$

where

$$
\begin{aligned}
\boldsymbol{a}(\theta) & =\left[a_{1}(\theta), \ldots, a_{L}(\theta)\right]^{T} \\
\boldsymbol{n}(t) & =\left[n_{1}(t), \ldots, n_{L}(t)\right]^{T}
\end{aligned}
$$

are the array response and sensor noise vectors, respectively. We also define the angular auto-correlation kernel for the kth source as the conjugate auto-correlation function between the signals distributed from the $k$ th source and impinging on the array from two different directions $\theta$ and $\theta^{\prime}$ as follows:

$$
p_{k k}\left(\theta, \theta^{\prime} ; \boldsymbol{\psi}_{k}, \boldsymbol{\psi}_{k}\right)=E\left\{s_{k}\left(\theta, \boldsymbol{\psi}_{k}, t\right) s_{k}^{*}\left(\theta^{\prime}, \boldsymbol{\psi}_{k}, t\right)\right\} .
$$

We assume that the sources are ID. This means that for each source, the signal components arriving from different scatterers are uncorrelated. Therefore, we have for the $k$ th source $[16,19,20]$ :

$$
p_{k k}\left(\theta, \theta^{\prime} ; \boldsymbol{\psi}_{k}, \boldsymbol{\psi}_{k}\right)=\sigma_{s_{k}}^{2} \rho_{k}\left(\theta, \boldsymbol{\psi}_{k}\right) \delta\left(\theta-\theta^{\prime}\right),
$$

where $\delta\left(\theta-\theta^{\prime}\right)$ is the Dirac delta-function and $\sigma_{s_{k}}^{2}$ is the power of the $k$ th source. Now, since the sources and noise are uncorrelated, then from (5) the covariance matrix of $\boldsymbol{x}(t)$ is explicitly given by:

$$
\begin{aligned}
\boldsymbol{R}_{x x}= & E\left\{\boldsymbol{x}(t) \boldsymbol{x}^{H}(t)\right\}, \\
= & \sum_{k=1}^{K} \sum_{k^{\prime}=1}^{K} \int_{-\pi / 2}^{\pi / 2} \int_{-\pi / 2}^{\pi / 2} p_{k k^{\prime}}\left(\theta, \theta^{\prime} ; \boldsymbol{\psi}_{k}, \boldsymbol{\psi}_{k^{\prime}}\right) \boldsymbol{a}(\theta) \boldsymbol{a}^{H}\left(\theta^{\prime}\right) d \theta d \theta^{\prime} \\
& +\sigma_{n}^{2} \boldsymbol{I}_{L},
\end{aligned}
$$

where $\sigma_{n}^{2}$ is the unknown noise power. Moreover, the function $p_{k k^{\prime}}\left(\theta, \theta^{\prime} ; \boldsymbol{\psi}_{k}, \boldsymbol{\psi}_{k^{\prime}}\right)$ stands for the angular crosscorrelation kernel between the $k$ th and $k^{\prime}$ th sources impinging on the array from the directions $\theta$ and $\theta^{\prime}$. It is defined as follows:

$$
p_{k k^{\prime}}\left(\theta, \theta^{\prime} ; \boldsymbol{\psi}_{k}, \boldsymbol{\psi}_{k^{\prime}}\right)=E\left\{s_{k}\left(\theta, \boldsymbol{\psi}_{k}, t\right) s_{k^{\prime}}^{*}\left(\theta^{\prime}, \boldsymbol{\psi}_{k^{\prime}}, t\right)\right\} .
$$

By assuming all the distributed sources to be mutually uncorrelated (i.e., every two different ID sources are uncorrelated), it follows that [19]:

$$
p_{k k^{\prime}}\left(\theta, \theta^{\prime} ; \boldsymbol{\psi}_{k}, \boldsymbol{\psi}_{k^{\prime}}\right)=p_{k k}\left(\theta, \theta^{\prime} ; \boldsymbol{\psi}_{k}, \boldsymbol{\psi}_{k}\right) \delta_{k k^{\prime}},
$$

where $\delta_{k k^{\prime}}$ is the Kronecker delta function defined as $\delta_{k k^{\prime}}=0$ for $k \neq k^{\prime}$ and 1 otherwise. Therefore, using (7) in (10), it follows that:

$$
p_{k k^{\prime}}\left(\theta, \theta^{\prime} ; \boldsymbol{\psi}_{k}, \boldsymbol{\psi}_{k^{\prime}}\right)=\sigma_{s_{k}}^{2} \rho_{k}\left(\theta, \boldsymbol{\psi}_{k}\right) \delta\left(\theta-\theta^{\prime}\right) \delta_{k k^{\prime}}
$$

Consequently, (8) reduces simply to:

$$
\boldsymbol{R}_{x x}=\sum_{k=1}^{K} \int_{-\pi / 2}^{\pi / 2} \sigma_{s_{k}}^{2} \rho_{k}\left(\theta, \psi_{k}\right) \boldsymbol{a}(\theta) \boldsymbol{a}^{H}(\theta) d \theta+\sigma_{n}^{2} \boldsymbol{I} .
$$

which can be further written in the following more succinct form:

$$
\boldsymbol{R}_{x x}=\sum_{k=1}^{K} \sigma_{s_{k}}^{2} \boldsymbol{R}_{s s}^{(k)}\left(\boldsymbol{\psi}_{k}\right)+\sigma_{n}^{2} \boldsymbol{I}_{L}
$$

where $\boldsymbol{R}_{s s}^{(k)}\left(\boldsymbol{\psi}_{k}\right)$ is the normalized noise-free autocovariance matrix of the $k$ th source given by:

$$
\boldsymbol{R}_{s s}^{(k)}\left(\boldsymbol{\psi}_{k}\right)=\int_{-\pi / 2}^{\pi / 2} \rho_{k}\left(\theta, \boldsymbol{\psi}_{k}\right) \boldsymbol{a}(\theta) \boldsymbol{a}^{H}(\theta) d \theta .
$$

Furthermore, we suppose that the sources often change their spatial positions resulting thereby in time-varying central DOAs $\overline{\boldsymbol{\theta}}(t)=\left[\bar{\theta}_{1}(t), \ldots, \bar{\theta}_{K}(t)\right]^{T}$. We also assume that the change in $\overline{\boldsymbol{\theta}}(t)$ is either zero or negligible over each interval of the DOA estimation step $[n T,(n+1) T]$, where $n=0,1, \ldots$ and $T$ represents the sampling period, i.e.,

$$
\overline{\boldsymbol{\theta}}(t) \approx \overline{\boldsymbol{\theta}}(n T), \quad \text { for } t \in[n T,(n+1) T], n=0,1, \ldots
$$

In principle, the motion of each source is slowly changing within a time increment $T$. Hence, we suppose that the change of the angular spread of each source is negligible during the tracking period. Then, the tracking problem aims at estimating $\overline{\boldsymbol{\theta}}(t), t=T, 2 T, \ldots$ from $N$ snapshots of array data measured within each time increment $T$ while keeping the angular spread the same during the tracking period.

\subsection{Derivation of the DOA tracking algorithm for incoherently distributed sources}

In this subsection, we propose a simple covariance-fittingbased DOA tracking scheme that tracks the central DOAs of multiple uncorrelated ID sources. The implementation of our new approach involves the two following functional structures:

- A covariance-fitting-based DOA estimator that exploits the central and noncentral moments of the 
source angular power densities in order to estimate the central DOAs over $[n T,(n+1) T]$ and

- A Kalman filter that tracks these DOAs during the tracking period.

In fact, to estimate the central DOAs of the uncorrelated ID sources over $[n T,(n+1) T]$, we propose to exploit the technique developed in [19] for constant DOAs. This method consists of approximating the covariance matrix using central and noncentral moments of the source angular power densities. Based on this approximation, these moments are estimated using a simple covariance fitting optimization technique. Finally, the central DOAs are obtained from the moment estimates. The advantage of this method is that it has a reduced computational cost and it addresses multiple sources with different angular power densities.

Once the estimated central DOAs are obtained, we then propose a Kalman-filtering-based tracking algorithm to model the dynamic property of directional changes for the sources. The KF ensures the association between the estimates made at different time points thanks to its predictability characteristic. Indeed, at each stage during the tracking process, the central DOAs predicted by the Kalman filter are used to smooth the central DOAs estimated via the covariance-fitting-based algorithm.

\subsubsection{Estimation of the central angles via the covariance-fitting-based algorithm}

In this subsection, we will recall briefly the covariance fitting optimization technique developed in [19] to estimate the central DOAs from $N$ samples received over the time interval $[n T,(n+1) T]$ over which the central DOAs are assumed to be invariant. Therefore, we will use $\bar{\theta}_{k}$ and $\psi_{k}$ instead of $\bar{\theta}_{k}(t)$ and $\psi_{k}(t)$, respectively.

As mentioned previously, the method developed in [19] is based on the approximation of the covariance matrix using central and noncentral moments of the sources' angular power densities. Actually, the $n$th noncentral moment of the angular power density of the $k$ th source around an arbitrary DOA $\tilde{\bar{\theta}}_{k}$ is defined as follows:

$$
M_{n k}\left(\tilde{\bar{\theta}}_{k}\right)=\int_{-\pi / 2}^{\pi / 2}\left(\theta-\tilde{\bar{\theta}}_{k}\right)^{n} \rho_{k}\left(\theta, \psi_{k}\right) d \theta .
$$

In (16), $\tilde{\bar{\theta}}_{k}$ represents a coarse initialization of the true central DOA $\bar{\theta}_{k}$ of the $k$ th source. Note here that if $\tilde{\bar{\theta}}_{k}=\bar{\theta}_{k}$, then $M_{n k}\left(\tilde{\bar{\theta}}_{k}\right)$ becomes the $n$th central moment, $M_{n k}\left(\bar{\theta}_{k}\right)$, of the $k$ th source angular power density.

It was proved in [19] that for the $k$ th source, the value of the first noncentral moment around an arbitrary DOA $\tilde{\bar{\theta}}_{k}$ determines the deviation of $\tilde{\bar{\theta}}_{k}$ with respect to the central DOA $\bar{\theta}_{k}$, i.e.,

$$
M_{1 k}\left(\tilde{\bar{\theta}}_{k}\right)=\bar{\theta}_{k}-\tilde{\bar{\theta}}_{k} \cdot
$$

Therefore, we conclude from (17) that in order to estimate the central DOA of the $k$ th source, one should calculate an estimate for the first noncentral moment of its angular power density. To do so, one use a simple covariance fitting optimization technique.

Covariance fitting optimization technique This technique is based on the approximation of the covariance matrix using a few noncentral moments of the source angular power densities. In fact, the covariance matrix $\boldsymbol{R}_{x x}$ can be approximated as follows [19]:

$$
\boldsymbol{R}_{x x} \simeq \tilde{\boldsymbol{R}}+\sigma_{n}^{2} \boldsymbol{I},
$$

where

$$
\tilde{\boldsymbol{R}}=\sum_{k=1}^{K} \sum_{r=0}^{R-1} \sigma_{s_{k}}^{2} M_{r k}\left(\tilde{\bar{\theta}}_{k}\right) C_{r k}\left(\tilde{\bar{\theta}}_{k}\right),
$$

with

$$
\boldsymbol{C}_{r k}\left(\tilde{\bar{\theta}}_{k}\right)=\left.\frac{1}{r !} \frac{\partial^{r} \boldsymbol{C}(\theta)}{\partial \theta^{r}}\right|_{\theta=\tilde{\bar{\theta}}_{k}} .
$$

Moreover, $R$ is an integer whose choice is discussed later, and $C(\theta)$ is defined as follows:

$$
\boldsymbol{C}(\theta)=\boldsymbol{a}(\theta) \boldsymbol{a}^{H}(\theta) .
$$

Equation 18 represents an approximation of the exact covariance matrix $\boldsymbol{R}_{x x}$ using $K R+1$ matrices. Note here that (19) is obtained using the $R$-term Taylor series approximation of $\boldsymbol{C}(\theta)$ around $\tilde{\bar{\theta}}_{k}$. In practical situations, $R$ should not be much larger than 1 . The approximation (18) is used to estimate the different noncentral moments and then derive the central DOAs from the first noncentral moment. In fact, we consider the following vectors:

$$
\begin{aligned}
\tilde{\overline{\boldsymbol{\theta}}} & =\left[\tilde{\bar{\theta}}_{1}, \tilde{\bar{\theta}}_{2}, \ldots, \tilde{\bar{\theta}}_{K}\right]^{T}, \\
\boldsymbol{m}(\tilde{\overline{\boldsymbol{\theta}}}) & =\left[\boldsymbol{m}_{1}^{T}\left(\tilde{\bar{\theta}}_{1}\right), \boldsymbol{m}_{2}^{T}\left(\tilde{\bar{\theta}}_{2}\right), \ldots, \boldsymbol{m}_{K}^{T}\left(\tilde{\bar{\theta}}_{K}\right), \sigma_{n}^{2}\right]^{T} \\
\boldsymbol{m}_{k}\left(\tilde{\bar{\theta}}_{k}\right) & =\sigma_{s_{k}}^{2}\left[1, M_{1 k}\left(\tilde{\bar{\theta}}_{k}\right), M_{2 k}\left(\tilde{\bar{\theta}}_{k}\right), \ldots, M_{(R-1) k}\left(\tilde{\bar{\theta}}_{k}\right)\right]^{T} .
\end{aligned}
$$

Assuming some initial value $\tilde{\overline{\boldsymbol{\theta}}}$ for the vector $\overline{\boldsymbol{\theta}}$, the noncentral moment vector $\boldsymbol{m}(\tilde{\overline{\boldsymbol{\theta}}})$ is obtained using the LS criterion as follows:

$$
\begin{aligned}
\widehat{\boldsymbol{m}}(\tilde{\overline{\boldsymbol{\theta}}}) & =\arg \min _{\boldsymbol{m}}\left\|\widehat{\boldsymbol{R}}_{x x}-\tilde{\boldsymbol{R}}-\sigma_{n}^{2} \boldsymbol{I}\right\|^{2}, \\
& =\arg \min _{\boldsymbol{m}} \operatorname{tr}\left\{\left(\widehat{\boldsymbol{R}}_{x x}-\left(\tilde{\boldsymbol{R}}+\sigma_{n}^{2} \boldsymbol{I}\right)\right)^{2}\right\} .
\end{aligned}
$$


where $\widehat{\boldsymbol{R}}_{x x}$ represents the sample covariance matrix defined as:

$$
\widehat{\boldsymbol{R}}_{x x}=\frac{1}{N} \sum_{t=1}^{N} \boldsymbol{x}(t) \boldsymbol{x}^{H}(t) .
$$

After straightforward manipulations as shown in [19], we find the following relation:

$$
\boldsymbol{Q}(\tilde{\overline{\boldsymbol{\theta}}}) \boldsymbol{m}(\tilde{\overline{\boldsymbol{\theta}}})=\boldsymbol{p}(\tilde{\overline{\boldsymbol{\theta}}}),
$$

where

$$
\begin{aligned}
& {[\boldsymbol{Q}(\tilde{\overline{\boldsymbol{\theta}}})]_{i j} }=\operatorname{tr}\left\{\boldsymbol{C}_{m l}\left(\tilde{\bar{\theta}}_{l}\right) \boldsymbol{C}_{r k}\left(\tilde{\bar{\theta}}_{k}\right)\right\}, \\
& {[\boldsymbol{p}(\tilde{\overline{\boldsymbol{\theta}}})]_{i}=\operatorname{tr}\left\{\boldsymbol{C}_{m l}\left(\tilde{\bar{\theta}}_{l}\right) \widehat{\boldsymbol{R}}_{x x}\right\}, }
\end{aligned}
$$

with $i=(l-1) R+m+1, j=(k-1) R+r+1(1 \leq l, k \leq$ $K, 0 \leq m, r<R)$.

Moreover, we have from [19]:

$$
\begin{aligned}
{[\boldsymbol{Q}(\tilde{\overline{\boldsymbol{\theta}}})]_{K R+1, j} } & =\operatorname{tr}\left(\boldsymbol{C}_{r k}\left(\tilde{\bar{\theta}}_{k}\right)\right), \\
{[\boldsymbol{Q}(\tilde{\overline{\boldsymbol{\theta}}})]_{i, K R+1} } & =\operatorname{tr}\left(\boldsymbol{C}_{r k}\left(\tilde{\bar{\theta}}_{k}\right)\right), \\
{[\boldsymbol{Q}(\tilde{\overline{\boldsymbol{\theta}}})]_{K R+1, K R+1} } & =L, \\
{[\boldsymbol{p}(\tilde{\overline{\boldsymbol{\theta}}})]_{K R+1} } & =\operatorname{tr}\left(\widehat{\boldsymbol{R}}_{x x}\right) .
\end{aligned}
$$

Therefore, from (27), the noncentral moments can be estimated as follows:

$$
\widehat{\boldsymbol{m}}(\tilde{\overline{\boldsymbol{\theta}}})=\boldsymbol{Q}^{-1}(\tilde{\overline{\boldsymbol{\theta}}}) \boldsymbol{p}(\tilde{\overline{\boldsymbol{\theta}}}),
$$

Note here that if the matrix $\boldsymbol{Q}(\tilde{\overline{\boldsymbol{\theta}}})$ is singular, we can replace its inverse by pseudoinverse.

Consequently, the central angles can be estimated as follows:

$$
\widehat{\bar{\theta}}_{k}=\widehat{M}_{1 k}\left(\tilde{\bar{\theta}}_{k}\right)+\tilde{\bar{\theta}}_{k}
$$

where $\widehat{M}_{1 k}\left(\tilde{\bar{\theta}}_{k}\right)$ is the first estimated noncentral moment of the angular density of the $k$ th source obtained from $\widehat{\boldsymbol{m}}(\tilde{\overline{\boldsymbol{\theta}}})$. Moreover, $\tilde{\bar{\theta}}_{k}$ is an arbitrary DOA that should be chosen sufficiently close to $\bar{\theta}_{k}$ to reduce the estimation errors.

To obtain a more accurate value of the estimated DOA, we replace $\tilde{\bar{\theta}}_{k}$ by $\widehat{\bar{\theta}}_{k}$ already calculated, and we solve again (27) to obtain the estimates of the central moments $\widehat{\boldsymbol{m}}(\widehat{\overline{\boldsymbol{\theta}}})$. Finally, we obtain a new value of the estimated central angle from the first estimated central moment $\widehat{M}_{1 k}\left(\widehat{\bar{\theta}}_{k}\right)$. This operation is repeated a few times.

Algorithm for the estimation of the central DOAs We can summarize the covariance-fitting-based algorithm to estimate the central DOAs at a fixed time as follows:

1. Compute the sample covariance matrix $\widehat{\boldsymbol{R}}_{x x}$ and specify the initial values of $\tilde{\bar{\theta}}_{k}, k=1,2, \ldots, K$;
2. Compute $\widehat{\boldsymbol{m}}(\tilde{\overline{\boldsymbol{\theta}}})$ from (34) and deduce $\widehat{M}_{1 m}\left(\tilde{\tilde{\bar{\theta}}_{k}}\right)$ using (23) and (24);

3. Compute $\widehat{\bar{\theta}}_{k}=\widehat{M}_{1 k}\left(\tilde{\bar{\theta}}_{k}\right)+\tilde{\bar{\theta}}_{k}$ and update $\tilde{\bar{\theta}}_{k}=\widehat{\bar{\theta}}_{k}$; and

4. Repeat steps 2 and 3 few times to obtain good estimates of the central DOAs.

These estimates will be treated as measurements and provided to the celebrated Kalman filter to track the DOAs.

\subsubsection{The KF tracking algorithm}

In order to model the dynamic directional changes for the moving sources, we propose a Kalman-filtering-based tracking algorithm. At each stage during the tracking process, instead of using the array output directly as a measurement, we propose to consider the current estimates of the central DOAs obtained with the covariance-fittingbased algorithm described in the previous subsection as measurements, predicted and updated via the Kalman state equation. To do so, we define the state vector for the $k$ th source as follows:

$$
\boldsymbol{y}_{k}(t)=\left[\bar{\theta}_{k}(t), \dot{\bar{\theta}}_{k}(t), \ddot{\bar{\theta}}_{k}(t)\right]^{T},
$$

where $\bar{\theta}_{k}(t)$ represents the source central DOA of the the $k$ th source, $\dot{\bar{\theta}}_{k}(t)$ is its first-order derivative reflecting its moving speed, and $\ddot{\bar{\theta}}_{k}(t)$ is its acceleration at time $t, k=$ $1,2, \ldots, K$. We model the dynamics and the measurement equations of the $k$ th source as follows:

$$
\begin{aligned}
\boldsymbol{y}_{k}(t+1) & =\boldsymbol{F} \boldsymbol{y}_{k}(t)+\boldsymbol{w}_{k}(t), \\
\hat{\bar{\theta}}_{k}(t) & =\boldsymbol{h} \boldsymbol{y}_{k}(t)+v_{k}(t),
\end{aligned}
$$

where $\boldsymbol{F}$ is the state transition matrix defined as follows:

$$
\boldsymbol{F}=\left(\begin{array}{ccc}
1 & T & \frac{1}{2} T^{2} \\
0 & 1 & T \\
0 & 0 & 1
\end{array}\right) .
$$

Moreover, $\boldsymbol{h}$ is the measurement matrix given by:

$$
\boldsymbol{h}=[1,0,0] \text {. }
$$

Furthermore, $\boldsymbol{w}_{k}(t)$ is the process noise vector caused by external circumstances like wind and bumps in the road. It is assumed to be Gaussian distributed with zero mean and covariance matrix:

$$
\boldsymbol{Q}_{k}=\sigma_{w}^{2}\left(\begin{array}{ccc}
\frac{T^{4}}{4} & \frac{T^{3}}{2} & \frac{T^{2}}{2} \\
\frac{T^{3}}{2} & T^{2} & T \\
\frac{T^{2}}{2} & T & 1
\end{array}\right) .
$$

In this paper, similar to $[23,31]$, we suppose that $\sigma_{w}^{2}$ is constant during the tracking period and small. Besides, $v_{k}(t)$ is the measurement noise which is supposed to be zero-mean with variance $\sigma_{v k}^{2}(t)$ and uncorrelated with $\boldsymbol{w}_{k}(t)$. 
As in the proposed KF tracking scheme, instead of using the array output directly as the measurement process, we use the most current data to form the DOA estimates via the covariance-fitting-based algorithm, the measurement noise is then due to the estimation inaccuracy of the covariance-fitting-based method.

To justify the use of the Kalman filter, we should show that the estimation error caused by the covariance-fittingbased algorithm is Gaussian distributed. In fact, we have from (35) that the estimated central DOA of the $k$ th source $\widehat{\bar{\theta}}_{k}$ is obtained as follows:

$$
\widehat{\bar{\theta}}_{k}=\widehat{M}_{1 k}\left(\tilde{\bar{\theta}}_{k}\right)+\tilde{\bar{\theta}}_{k} \text {, }
$$

where $\widehat{M}_{1 k}\left(\tilde{\bar{\theta}}_{k}\right)$ represents the $((k-1) R+2)$ th element of $\widehat{\boldsymbol{m}}(\tilde{\overline{\boldsymbol{\theta}}})$. Then, we have the following estimation error that also represents the measurement noise of the $k$ th source:

$$
\begin{aligned}
v_{k}(t) & =\widehat{\bar{\theta}}_{k}-\bar{\theta}_{k}, \\
& =\widehat{M}_{1 k}\left(\tilde{\bar{\theta}}_{k}\right)+\left(\tilde{\bar{\theta}}_{k}-\bar{\theta}_{k}\right), \\
& =\widehat{M}_{1 k}\left(\tilde{\bar{\theta}}_{k}\right)+\Delta \bar{\theta}_{k},
\end{aligned}
$$

in which $\Delta \bar{\theta}_{k}=\tilde{\bar{\theta}}_{k}-\bar{\theta}_{k}$ is deterministic and is negligible $\left(\tilde{\bar{\theta}}_{k}\right.$ should be chosen sufficiently close to $\bar{\theta}_{k}$ and then $\left.\Delta \bar{\theta}_{k} \rightarrow 0\right)$. Therefore, to show that the estimation error generated from central DOA estimation is Gaussian, we should prove that $\widehat{M}_{1 k}\left(\tilde{\bar{\theta}}_{k}\right)$ is Gaussian distributed. In fact, we have that $\widehat{M}_{1 k}\left(\tilde{\bar{\theta}}_{k}\right)$ is the $((k-1) R+2)$ th element of $\widehat{\boldsymbol{m}}(\tilde{\overline{\boldsymbol{\theta}}})$. Moreover, we have from (34) that:

$$
\widehat{\boldsymbol{m}}(\tilde{\overline{\boldsymbol{\theta}}})=\boldsymbol{Q}^{-1}(\tilde{\overline{\boldsymbol{\theta}}}) \boldsymbol{p}(\tilde{\overline{\boldsymbol{\theta}}}) .
$$

Otherwise, we verify from (28), (30), (31), and (32) that $\boldsymbol{Q}^{-1}(\tilde{\overline{\boldsymbol{\theta}}})$ depends only on $\boldsymbol{C}_{i j}\left(\tilde{\bar{\theta}}_{k}\right)$ for fixed values of $i$ and $j$. Then, this term is deterministic. While we have from (29) and (33), $\boldsymbol{p}(\tilde{\overline{\boldsymbol{\theta}}})$ depends on the estimated covariance matrix:

$$
\widehat{\boldsymbol{R}}_{x x}=\frac{1}{N} \sum_{t=1}^{N} \boldsymbol{x}(t) \boldsymbol{x}^{H}(t) .
$$

Therefore, we can define a functional relation linking the estimate $\widehat{M}_{1 k}\left(\tilde{\bar{\theta}}_{k}\right)$ to the statistics $\widehat{\boldsymbol{R}}_{x x}$ as follows:

$$
\widehat{M}_{1 k}\left(\tilde{\bar{\theta}}_{k}\right)=\operatorname{alg}\left(\widehat{\boldsymbol{R}}_{x x}\right) \text {. }
$$

Otherwise, the snapshots $x(t)_{t=1, \ldots, N}$ are random and mutually independent. Then, using the classical central limit theorem [32], we have for a large number of snapshots $N$ that $\widehat{\boldsymbol{R}}_{x x}$ follows a normal distribution. We can then conclude from [33] that $\widehat{M}_{1 k}\left(\tilde{\bar{\theta}}_{k}\right)=\operatorname{alg}\left(\widehat{\boldsymbol{R}}_{x x}\right)$ is asymptotically Gaussian distributed. Consequently, the measurement noise is Gaussian. We will also verify in subsection 1.4 that the estimation error of the covariancefitting-based algorithm is Gaussian distributed.
In practice, the variance of the measurement noise for the $k$ th source, $\sigma_{v k}^{2}(t)$, is basically determined by the variance of the estimation error of the covariance-fittingbased algorithm over each time interval $[n T,(n+1) T]$. Note here that Rao et al. suggested in [34] in a similar context that the variances of the measurement noise can be approximated by the diagonal entries of the CramérRao bounds (CRBs) for large number of samples. Moreover, Sanchez-Araujo and Marcos proposed in [23] to utilize the asymptotic (for $N>1$ ) theoretical variance expression of the considered DOA estimation method to calculate the variance of the measurement noise. Therefore, the variances of the measurement noise due to the estimation error of the covariance-fitting-based algorithm $\left\{\sigma_{v k}^{2}(t)\right\}_{k=1}^{K}$ should be calculated at each time interval $[n T,(n+1) T]$. Moreover, these variances as suggested by Rao et al. can be theoretically approximated by the diagonal entries of the CRBs derived in [35] for ID sources for a large number of samples. In fact, considering the unknown parameter vector, $\boldsymbol{v}$, as follows:

$$
\begin{aligned}
\boldsymbol{v} & =\left[\overline{\boldsymbol{\theta}}^{T}, \boldsymbol{\sigma}^{T}, \sigma_{n}^{2}\right]^{T}, \\
& =\left[\overline{\boldsymbol{\theta}}^{T}, \boldsymbol{\xi}^{T}\right]^{T},
\end{aligned}
$$

in which the vector $\overline{\boldsymbol{\theta}}$ contains the central DOAs of the ID sources, the vector $\sigma$, is the vector of the unknown angular spreads and $\sigma_{n}^{2}$ is the unknown noise power. Therefore, the associated FIM can be written as:

$$
\boldsymbol{I}(\boldsymbol{v})=\left(\begin{array}{cc}
\boldsymbol{I}_{\overline{\boldsymbol{\theta}}, \overline{\boldsymbol{\theta}}} & \boldsymbol{I}_{\xi, \overline{\boldsymbol{\theta}}} \\
\boldsymbol{I}_{\overline{\boldsymbol{\theta}}, \bar{\xi}} & \boldsymbol{I}_{\xi, \xi}
\end{array}\right)
$$

whose $i j$ th entry is expressed as:

$$
[\boldsymbol{I}]_{i j}=N \operatorname{tr}\left\{\frac{\partial \boldsymbol{R}_{x x}}{\partial v_{i}} \boldsymbol{R}_{x x}^{-1} \frac{\partial \boldsymbol{R}_{x x}}{\partial v_{j}} \boldsymbol{R}_{x x}^{-1}\right\},
$$

with $v_{i}$ is the $i$ th element of $\boldsymbol{v}$. The CRB of the unknown parameter vector, $\boldsymbol{v}$, is defined as follows:

$$
\operatorname{CRB}(\boldsymbol{v})=\boldsymbol{I}^{-1}(\boldsymbol{v}),
$$

As we are interested to the CRB of the central DOAs $\overline{\boldsymbol{\theta}}$ which we denote as $\operatorname{CRB}(\overline{\boldsymbol{\theta}})$, we use the inversion of block matrices of Lemma [36] to obtain the following expression of $\operatorname{CRB}(\overline{\boldsymbol{\theta}})$ :

$$
\mathrm{CRB}(\overline{\boldsymbol{\theta}})=\left(\boldsymbol{I}_{\overline{\boldsymbol{\theta}}, \overline{\boldsymbol{\theta}}}-\boldsymbol{I}_{\xi, \overline{\boldsymbol{\theta}}}^{T} \boldsymbol{I}_{\xi, \xi}^{-1} \boldsymbol{I}_{\xi, \overline{\boldsymbol{\theta}}}\right)^{-1}
$$

Then, the variances of the measurement noise $\left\{\sigma_{v k}^{2}(t)\right\}_{k=1}^{K}$ can be approximated by the diagonal entries of $\operatorname{CRB}(\overline{\boldsymbol{\theta}})$ at each time interval $[n T,(n+1) T]$ for large number of samples.

We assume that the motion of each source is mutually independent. We define the total state vector of the $K$ moving sources as $y(t)=\left[\boldsymbol{y}_{1}^{T}(t), \ldots, \boldsymbol{y}_{K}^{T}(t)\right]^{T}$. Then, from 
(37) and (38), the dynamic and measurement models are given by the following equations:

$$
\begin{aligned}
\boldsymbol{y}(t+1) & =\overline{\boldsymbol{F}} \boldsymbol{y}(t)+\boldsymbol{w}(t), \\
\hat{\overline{\boldsymbol{\theta}}}(t) & =\overline{\boldsymbol{H}} \boldsymbol{y}(t)+\boldsymbol{v}(t),
\end{aligned}
$$

where

$$
\begin{aligned}
\overline{\boldsymbol{F}} & =\left(\begin{array}{ccccc}
1 & T & \frac{1}{2} T^{2} & \cdots & 0 \\
0 & 1 & T & \cdots & 0 \\
0 & 0 & 1 & \cdots & 0 \\
\vdots & & \ddots & & \vdots \\
0 & \cdots & 1 & T & \frac{1}{2} T^{2} \\
0 & \cdots & 0 & 1 & T \\
0 & \cdots & 0 & 0 & 1
\end{array}\right), \\
\overline{\boldsymbol{H}} & =\left(\begin{array}{ccccc}
1 & 0 & 0 & \cdots & 0 \\
\vdots & & \ddots & & \vdots \\
0 & \cdots & 1 & 0 & 0
\end{array}\right)
\end{aligned}
$$

In addition, $\boldsymbol{w}(t)=\left[\boldsymbol{w}_{1}^{T}(t), \ldots, \boldsymbol{w}_{K}^{T}(t)\right]^{T}$ and $\boldsymbol{v}(t)=$ $\left.\left[v_{1}(t), \ldots, v_{K}(t)\right)\right]^{T}$ are Gaussian distributed with zeromean vectors and covariances $\overline{\boldsymbol{Q}}$ and $\boldsymbol{R}(t)$, respectively, defined as follows:

$$
\begin{aligned}
& \overline{\mathbf{Q}}=\sigma_{w}^{2}\left(\begin{array}{ccccc}
\frac{T^{4}}{4} & \frac{T^{3}}{2} & \frac{T^{2}}{2} & \cdots & 0 \\
\frac{T^{3}}{2} & T^{2} & T & \cdots & 0 \\
\frac{T^{2}}{2} & T & 1 & \cdots & 0 \\
\vdots & & \ddots & & \vdots \\
0 & \cdots & \frac{T^{4}}{4} & \frac{T^{3}}{2} & \frac{T^{2}}{2} \\
0 & \cdots & \frac{T^{3}}{2} & T^{2} & T \\
0 & \cdots & \frac{T^{2}}{2} & T & 1
\end{array}\right), \\
& \boldsymbol{R}(t)=\operatorname{diag}\left(\left[\sigma_{v 1}^{2}(t), \ldots, \sigma_{v K}^{2}(t)\right]\right) .
\end{aligned}
$$

The proposed tracking algorithm of the central DOAs $\overline{\boldsymbol{\theta}}$ can then be summarized as follows.

- Initially, the covariance-fitting-based algorithm is used twice to obtain two angle estimates $\hat{\overline{\boldsymbol{\theta}}}(-1)$ and $\hat{\overline{\boldsymbol{\theta}}}(0)$ at $t=0$. Then, the initial state vector is $\hat{\boldsymbol{y}}(0 \mid 0)=\left[\hat{\bar{\theta}}_{1}(0),\left(\hat{\bar{\theta}}_{1}(0)-\hat{\bar{\theta}}_{1}(-1)\right) / T, 0, \ldots, \hat{\bar{\theta}}_{K}(0)\right.$, $\left.\left(\hat{\bar{\theta}}_{K}(0)-\hat{\bar{\theta}}_{K}(-1)\right) / T, 0\right]^{T}$.

- Calculate the initial variances of the measurement noise $\left\{\sigma_{v k}^{2}(0)\right\}_{k=1}^{K}$ which represent the variances of the estimation error of the covariance-fitting-based algorithm at $t=0$. These variances can also be obtained from the diagonal entries of (54) for a large number of samples.
- Calculate the covariance matrix of the initial state vector $\hat{\boldsymbol{y}}(0 \mid 0)$ as follows:

$$
\begin{aligned}
\boldsymbol{P}(0 \mid 0) & =\operatorname{diag}\left(\left[\sigma_{v 1}^{2}(0), \ldots, \sigma_{v K}^{2}(0)\right]\right) \otimes\left(\begin{array}{ccc}
1 & \frac{1}{T} & 0 \\
\frac{1}{T} & \frac{2}{T^{2}} & 0 \\
0 & 0 & 0
\end{array}\right), \\
& =\boldsymbol{R}(0) \otimes\left(\begin{array}{ccc}
1 & \frac{1}{T} & 0 \\
\frac{1}{T} & \frac{2}{T^{2}} & 0 \\
0 & 0 & 0
\end{array}\right) .
\end{aligned}
$$

- For $t=1,2, \ldots$, we do the following steps

- Prediction of DOA angles: obtain the predicted estimates $\hat{\boldsymbol{y}}(t \mid t-1)$ of the state vector $\boldsymbol{y}(t)$ from the existing estimates $\hat{\boldsymbol{y}}(t-1 \mid t-1)$ available at time $t-1$ and its covariance matrix $\boldsymbol{P}(t \mid t-1)$ by the equations:

$$
\begin{aligned}
\hat{\boldsymbol{y}}(t \mid t-1) & =\overline{\boldsymbol{F}} \hat{\boldsymbol{y}}(t-1 \mid t-1), \\
\boldsymbol{P}(t \mid t-1) & =\overline{\boldsymbol{F}} \boldsymbol{P}(t-1 \mid t-1) \overline{\boldsymbol{F}}^{T}+\overline{\boldsymbol{Q}} .
\end{aligned}
$$

Then, we obtain the predicted central DOAs vector $\hat{\overline{\boldsymbol{\theta}}}(t \mid t-1)$ from $\hat{\boldsymbol{y}}(t \mid t-1)$ as follows:

$$
\hat{\boldsymbol{\theta}}(t \mid t-1)=\overline{\boldsymbol{H}} \hat{\boldsymbol{y}}(t \mid t-1) .
$$

- Central DOA estimation via the covariance-fitting-based method: we estimate the central angles $\hat{\overline{\boldsymbol{\theta}}}(t)$ via the covariance-fitting-based algorithm as described in the previous subsection.

- Updating the covariance matrix of the measurement noise vector $\boldsymbol{R}(t)$ : we calculate the variances of the measurement noise $\left\{\sigma_{v k}^{2}(t)\right\}_{k=1}^{K}$, and we then deduce the covariance matrix $\boldsymbol{R}(t)=\operatorname{diag}\left(\left[\sigma_{v 1}^{2}(t), \ldots, \sigma_{v K}^{2}(t)\right]\right)$.

- Updating the estimated DOA angles: in this last step, we aim to find the estimate $\hat{\boldsymbol{y}}(t \mid t)$ of the state vector $\boldsymbol{y}(t)$. Therefore, we should first estimate the innovation errors $\delta \overline{\boldsymbol{\theta}}$ defined as:

$$
\delta \overline{\boldsymbol{\theta}}(t)=\hat{\overline{\boldsymbol{\theta}}}(t)-\hat{\overline{\boldsymbol{\theta}}}(t \mid t-1) .
$$

Because the motion of each source is mutually independent, the innovation errors vector $\delta \boldsymbol{\theta}(t)$ is zero mean with covariance $\overline{\boldsymbol{H}} \boldsymbol{P}(t \mid t-1) \overline{\boldsymbol{H}}^{T}+\boldsymbol{R}(t)$. After the determination of $\delta \overline{\boldsymbol{\theta}}(t)$, we update the state vector by the following equation:

$$
\hat{\boldsymbol{y}}(t \mid t)=\hat{\boldsymbol{y}}(t \mid t-1)+\boldsymbol{G}(t) \delta \overline{\boldsymbol{\theta}}(t),
$$

where $\boldsymbol{G}(t)$ is the Kalman gains vector at time $t$ given by:

$$
\boldsymbol{G}(t)=\boldsymbol{P}(t \mid t-1) \overline{\boldsymbol{H}}^{T}\left[\overline{\boldsymbol{H}} \boldsymbol{P}(t \mid t-1) \overline{\boldsymbol{H}}^{T}+\boldsymbol{R}(t)\right]^{-1} .
$$


The covariance matrix of $\hat{\boldsymbol{y}}(t \mid t)$ is obtained as:

$$
\boldsymbol{P}(t \mid t)=(I-\boldsymbol{G}(t) \overline{\boldsymbol{H}}) \boldsymbol{P}(t \mid t-1) .
$$

Finally, we obtain the updated central DOA vector $\hat{\overline{\boldsymbol{\theta}}}(t \mid t)$ from $\hat{\boldsymbol{y}}(t \mid t)$ as follows:

$$
\hat{\overline{\boldsymbol{\theta}}}(t \mid t)=\overline{\boldsymbol{H}} \hat{\boldsymbol{y}}(t \mid t) .
$$

\subsection{Simulation results}

In this subsection, we will present some figures to illustrate the effectiveness of our proposed algorithm in different scenarios and compare it to the method derived in [28] (referred to as FAPI-TLS-ESPRIT method). It will be seen that our new method outperforms the FAPI-TLS-ESPRIT method.

Throughout this section, we consider a zero-mean, Gaussian distributed, spatially and temporally white noise. We also consider a uniform linear array of 11 sensors separated by a half-wavelength $\lambda / 2$. The sources are tracked over an interval of $40 \mathrm{~s}$ with $T=1 \mathrm{~s}$. During each 1 -s interval, $N=500$ snapshots of sensor data are generated and used to estimate the central DOAs via the covariancefitting-based algorithm with $R=3$. In addition, three iterations of steps 2 and 3 of this algorithm are used in all our examples. Moreover, 100 independent simulation runs have been performed to obtain each simulated central DOA vector. To track the DOAs, we assume that the variance of the process noise in the KF is constant and equal to $\sigma_{w}^{2}=0.0001$ while the variances of the measurement noise $\left\{\sigma_{v k}^{2}(t)\right\}_{k=1}^{K}$ are calculated at each time interval $[n T,(n+1) T]$ as explained in the previous section.

To simulate the FAPI-TLS-ESPRIT method in a proper way, we consider two identical subarrays of 11 sensors with a half-wavelength interelement spacing. The intersubarray displacement is assumed to be $\delta=\lambda / 10$. Moreover, to estimate the signal subspace via the FAPI algorithm, we choose the forgetting factor $\beta=0.985$.

We first consider two narrowband ID sources with different distributions. The first one is assumed to be Gaussian distributed (GID) with central DOA $\bar{\theta}_{1}=-10^{\circ}$ and angular spread $\sigma_{1}=1^{\circ}$ and the second one is Laplacian distributed (LID) with central angle $\bar{\theta}_{2}=30^{\circ}$ and angular spread $\sigma_{2}=2.5^{\circ}$. As the case of low SNR values represents the most challenging scenario in the literature, we will consider particularly in most of our simulations the case of low SNR levels. Therefore, we suppose that the signal-to-noise ratio is $\mathrm{SNR}=$ $0 \mathrm{~dB}$. To justify the use of the Kalman filter, we will verify through Fig. 1a, b that the estimation error of the covariance-fitting-based algorithm follows Gaussian distribution.

Figures 2, 3, and 4 show the tracking performances of our proposed algorithm and the FAPI-TLS-ESPRIT algorithm. Figure 2 displays the tracking trajectories of the central DOAs estimated by the proposed algorithm and the FAPI-TLS-ESPRIT method comparing with the true trajectories, while Fig. 3 exhibits the estimation errors
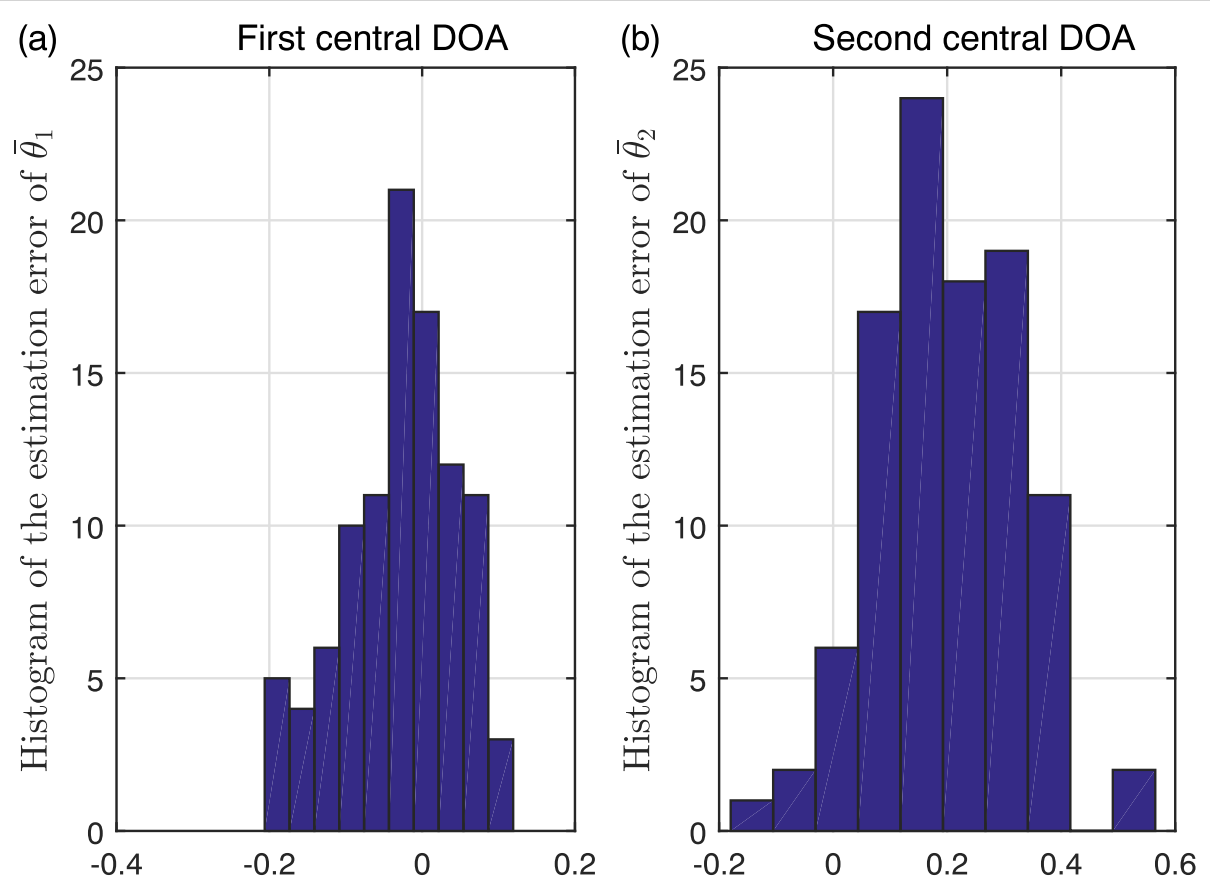

Fig. 1 Histogram of the estimation errors of the central DOAs $-10^{\circ}(\mathbf{a})$ and $30^{\circ}(\mathbf{b})$ at $\mathrm{SNR}=0 \mathrm{~dB}$ 


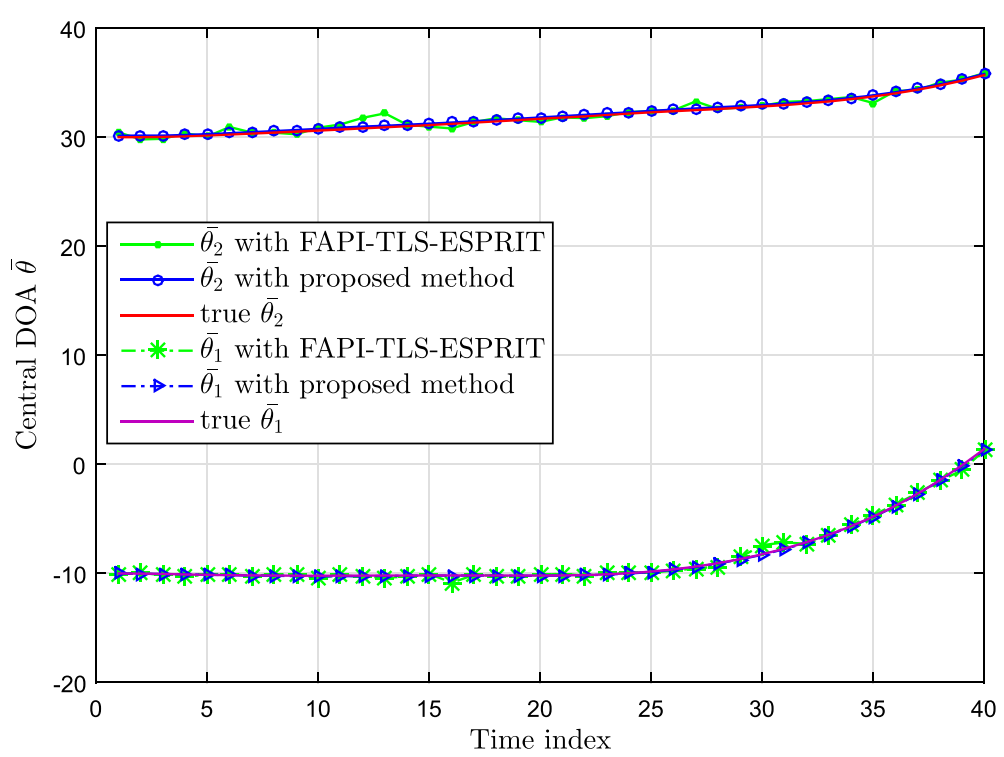

Fig. 2 Tracking trajectories using the proposed algorithm and the FAPI-TLS-ESPRIT method for two GID and LID sources with central DOAs $-10^{\circ}$ and $30^{\circ}$ at $\mathrm{SNR}=0 \mathrm{~dB}$ and $N=500$

of the two methods. The estimation error is defined as the absolute value of the difference between the mean value of the estimated DOA and the true DOA. Moreover, Fig. 4 shows the variances of the estimated central DOAs obtained for the two methods. We show from these figures that our method can accurately track each source trajectory. Moreover, it outperforms the FAPI-TLS-ESPRIT method. In fact, it can be seen from Fig. 2 that the tracking trajectory of the central DOAs estimated with the
FAPI-TLS-ESPRIT method deviates in some points from the true values. However, our proposed method can exactly and precisely estimate the true values of the central DOAs throughout the tracking period. This result is more illustrated by Figs. 3 and 4. Indeed, we notice from these figures that the estimation errors and the variances of the estimated central DOAs of our proposed method are greatly lower than those made by the other method. This estimation error and these variances are also stable with

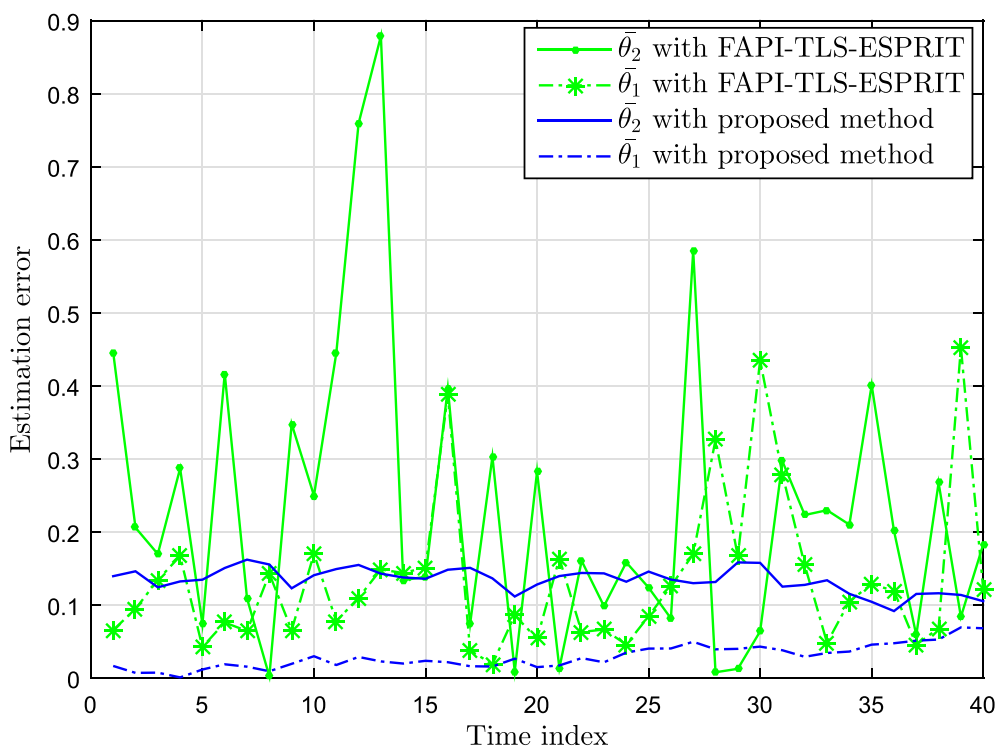

Fig. 3 Estimation errors made by the proposed algorithm and the FAPI-TLS-ESPRIT method for two GID and LID sources with central DOAs $-10^{\circ}$ and $30^{\circ}$ at $\mathrm{SNR}=0 \mathrm{~dB}$ and $N=500$ 


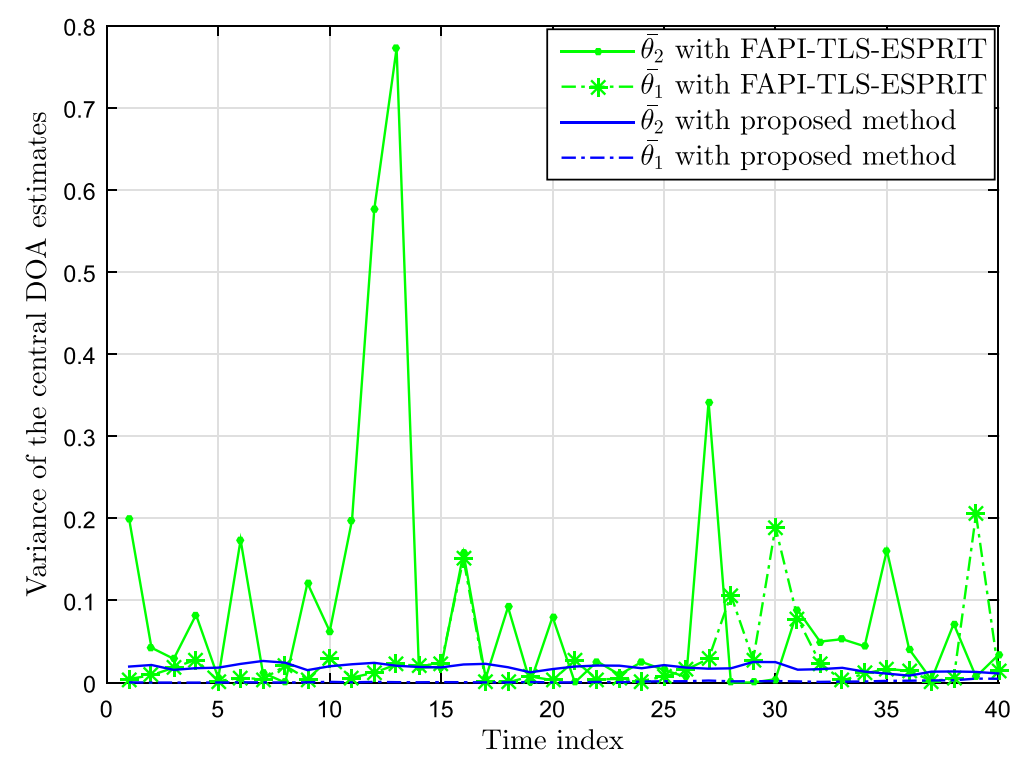

Fig. 4 Variances of the estimated central DOAs of the proposed algorithm and the FAPI-TLS-ESPRIT method for two GID and LID sources with central DOAs $-10^{\circ}$ and $30^{\circ}$ at $\mathrm{SNR}=0 \mathrm{~dB}$ and $N=500$

our algorithm whereas they vary randomly and present many peaks with the FAPI-TLS-ESPRIT algorithm. Now, we consider the same scenario of the first example, but we reduce the number of snapshots $N$ at every iteration of the tracking process to $N=100$ and then to $N=50$. Figures 5 and 6 present the variances of the estimated central DOAs obtained for the two methods for $N=100$ and $N=50$, respectively. We show from these figures that the variances of the estimated central DOAs of our proposed method are low. Moreover, they are significantly lower than those made by the FAPI-TLS-ESPRIT method. This proves that our proposed algorithm has also good performances even with these moderate numbers of snapshots. However, when comparing these figures with Fig. 4, we can see that the variances of the central DOAs estimates using our proposed algorithm are slightly more stable when $N=500$ than $N=100$ and $N=50$.

After, we consider two ID sources with the same distributions as in the previous example but with different location parameters. The first one is GID with central

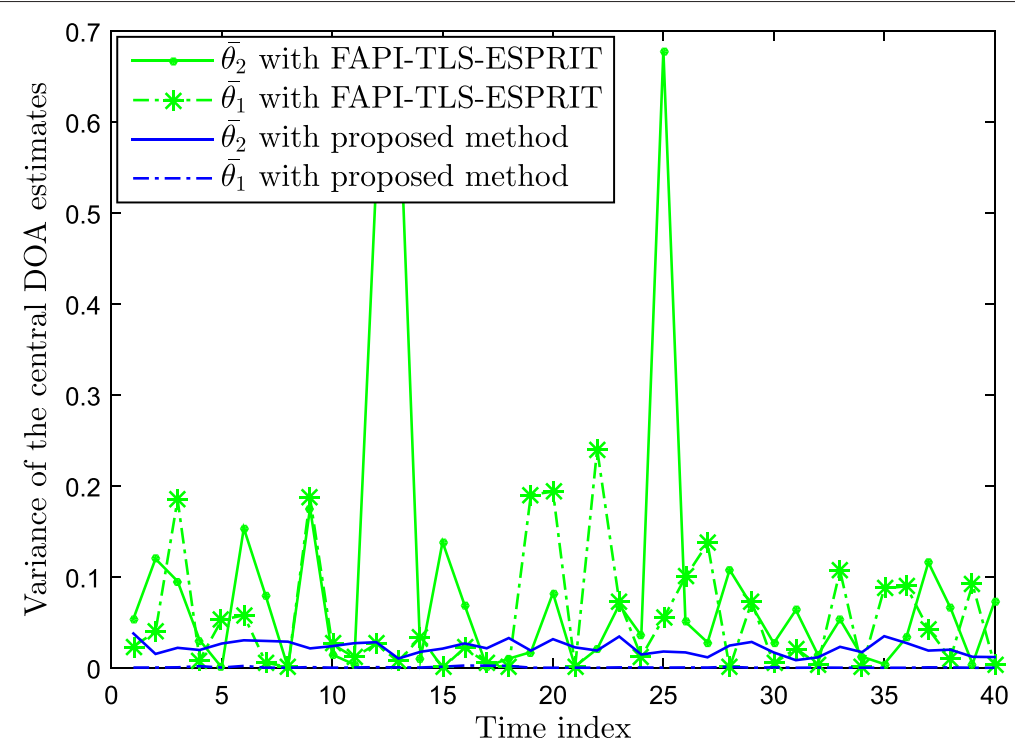

Fig. 5 Variances of the estimated central DOAs of the proposed algorithm and the FAPI-TLS-ESPRIT method for two GID and LID sources with central $\mathrm{DOAs}-10^{\circ}$ and $30^{\circ}$ at $\mathrm{SNR}=0 \mathrm{~dB}$ and $N=100$ 


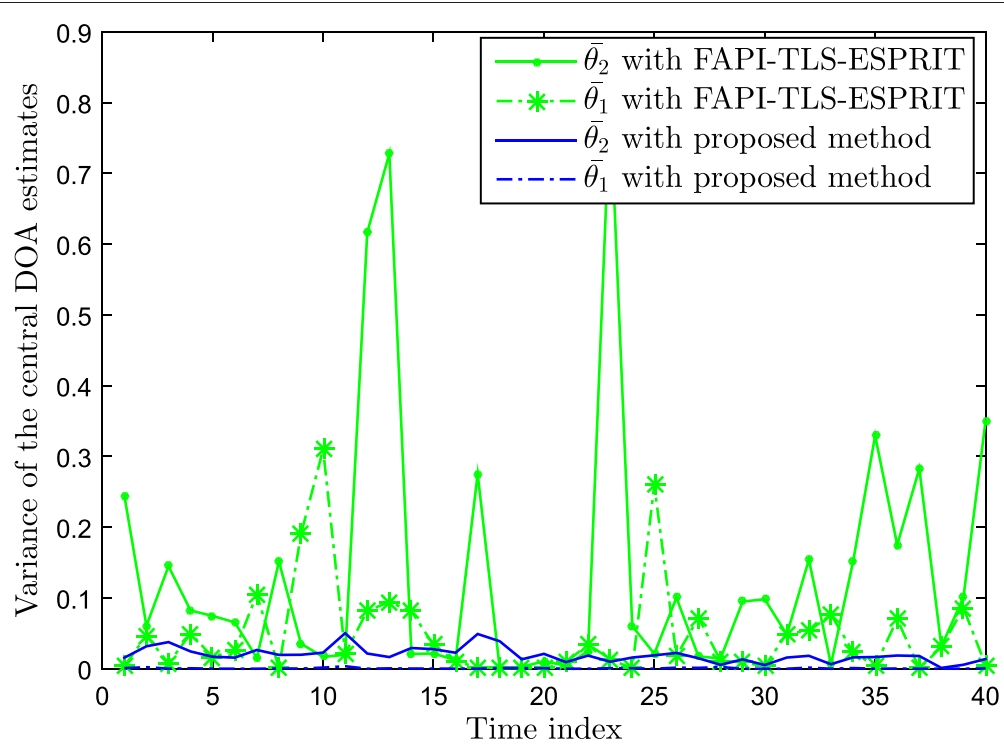

Fig. 6 Variances of the estimated central DOAs of the proposed algorithm and the FAPI-TLS-ESPRIT method for two GID and LID sources with central DOAs $-10^{\circ}$ and $30^{\circ}$ at $S N R=0 \mathrm{~dB}$ and $N=50$

DOA $\bar{\theta}_{1}=10^{\circ}$ and angular spread $\sigma_{1}=1.5^{\circ}$ and the second one is LID with central DOA $\bar{\theta}_{2}=30^{\circ}$ and angular spread $\sigma_{2}=2.5^{\circ}$. We also suppose that the $\mathrm{SNR}=0 \mathrm{~dB}$ and the number of snapshots is $N=500$. Figures 7,8 , and 9 present the tracking trajectories, the estimation errors, and the variances of the estimated DOAs of our proposed algorithm and the FAPI-TLS-ESPRIT algorithm, respectively.
These figures illustrate the result proved in the first example. The proposed algorithm offers an excellent DOA tracking performance and outperforms the FAPI-TLSESPRIT method at low SNR values. Moreover, when we compare the estimation error of the first central DOA made by our proposed method in Fig. 3 to that in Fig. 8, we note that this error in the first case is lower than the corresponding error in the second case. The same holds for

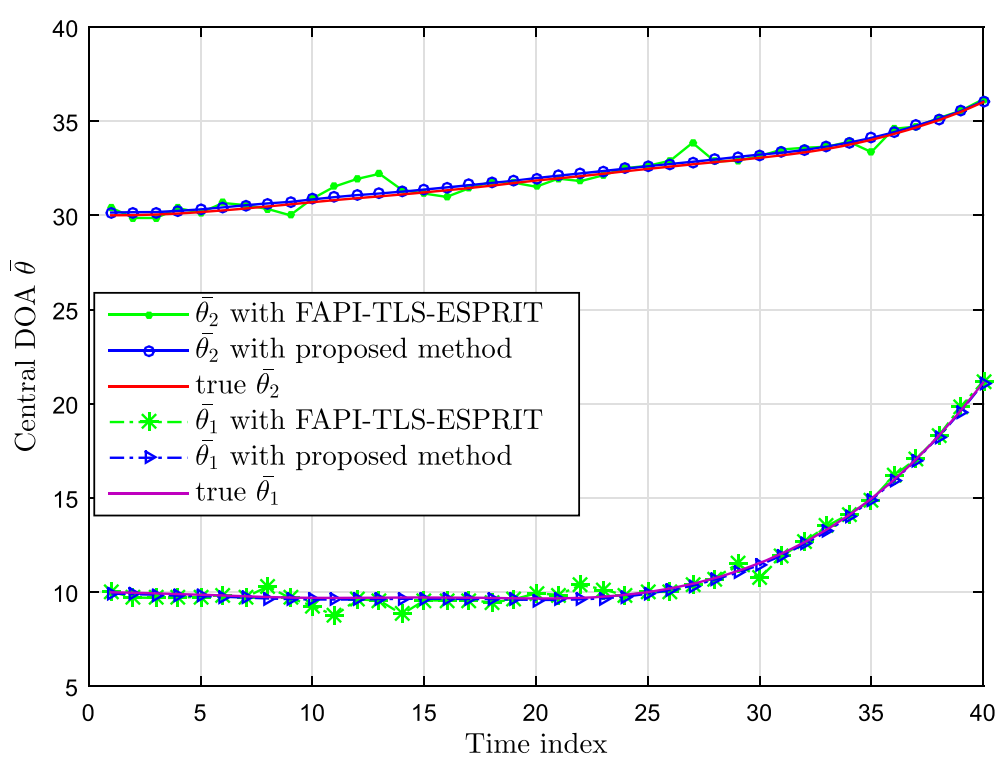

Fig. 7 Tracking trajectories using the proposed algorithm and the FAPI-TLS-ESPRIT method for two GID and LID sources with central DOAs $10^{\circ}$ and $30^{\circ}$ at $\mathrm{SNR}=0 \mathrm{~dB}$ 


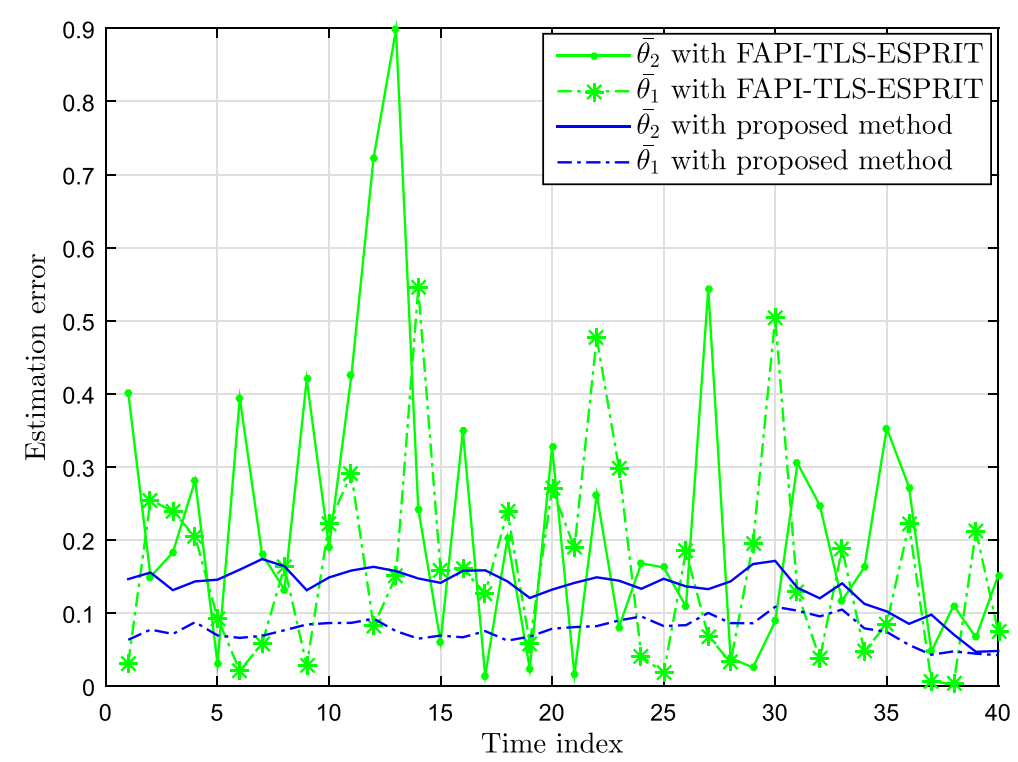

Fig. 8 Estimation errors made by the proposed algorithm and the FAPI-TLS-ESPRIT method for two GID and LID sources with central DOAs $10^{\circ}$ and $30^{\circ}$ at $S N R=0 \mathrm{~dB}$

the variance of the first estimated central DOA. In fact, we see clearly from Fig. 4 that, in the first case, this variance is too low. This can be explained by the fact that in the first case, the sources are more spaced than in the second case. Indeed, in Figs. 3 and 4, the central DOAs are $\bar{\theta}_{1}=-10^{\circ}$ and $\bar{\theta}_{2}=30^{\circ}$ while in Figs. 8 and 9 the central DOAs are $\bar{\theta}_{1}=10^{\circ}$ and $\bar{\theta}_{2}=30^{\circ}$. Therefore, we can conclude that the more the sources are spaced, the more the proposed method can exactly track the central DOAs.
Now, we consider the same narrowband ID sources as in the second example. But we suppose that SNR = $10 \mathrm{~dB}$. The tracking trajectories, the estimation errors, and the variances of the central DOAs estimated by the two methods are presented in Figs. 10, 11, and 12, respectively.

We see from Fig. 10 that the two methods can exactly track the central DOAs at high SNR values. Moreover, we note from Figs. 11 and 12 that despite the random variation of the estimation errors and the variances of the

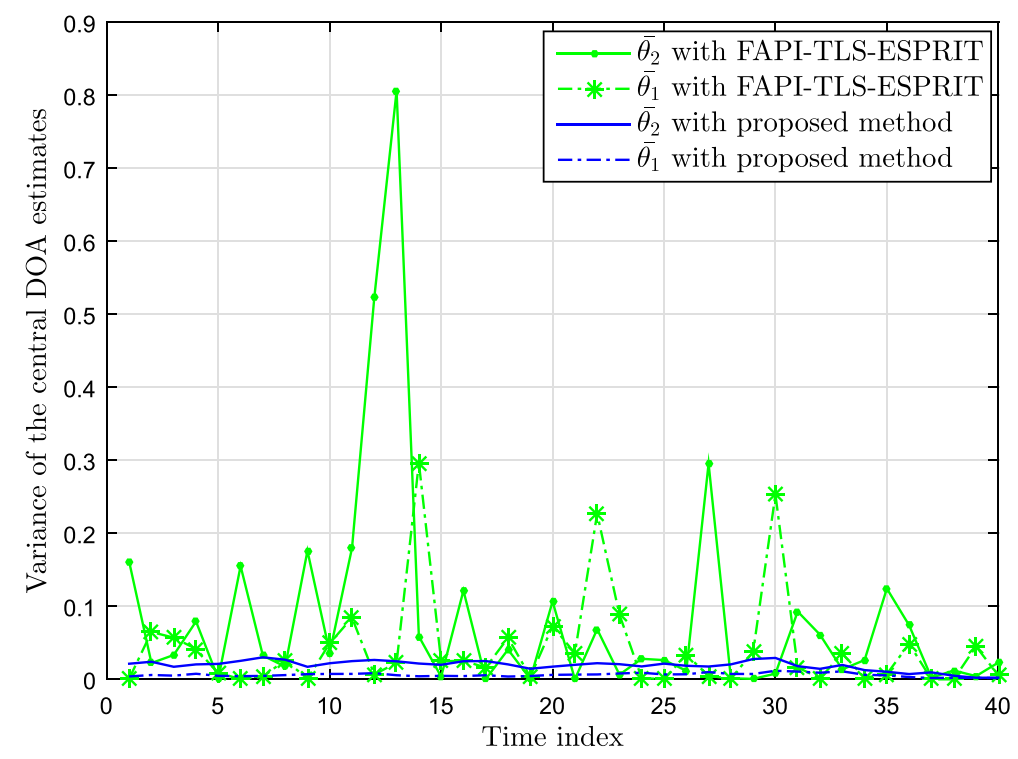

Fig. 9 Variances of the estimated central DOAs of the proposed algorithm and the FAPI-TLS-ESPRIT method for two GID and LID sources with central $\mathrm{DOAs} 10^{\circ}$ and $30^{\circ}$ at $\mathrm{SNR}=0 \mathrm{~dB}$ 


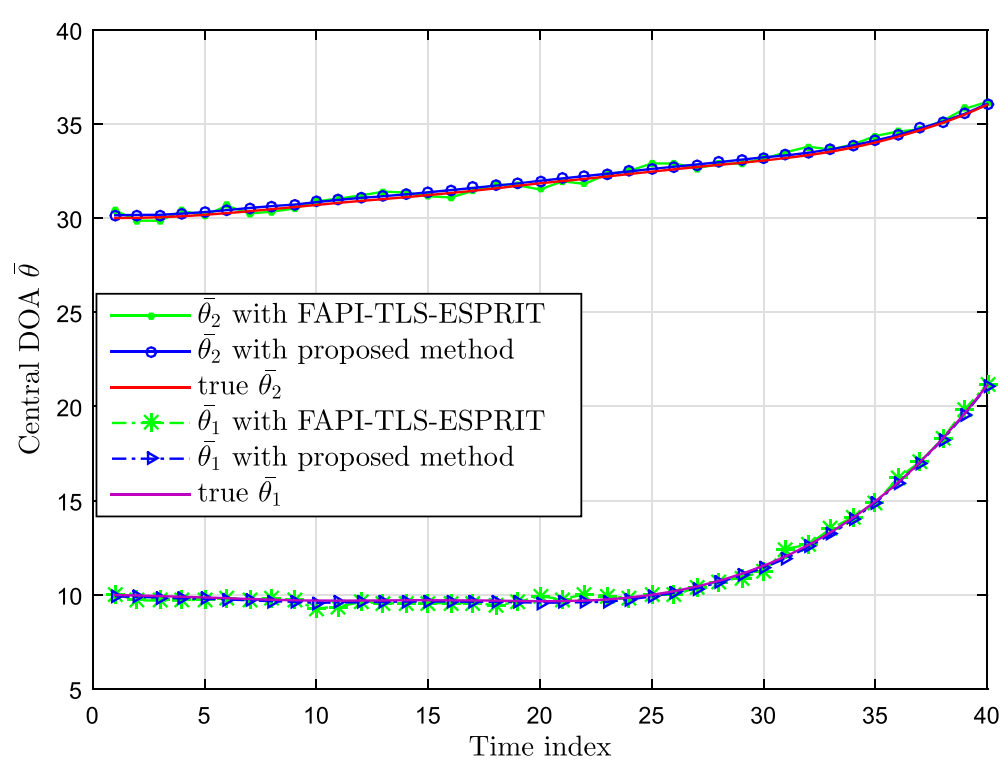

Fig. 10 Tracking trajectories using the proposed algorithm and the FAPI-TLS-ESPRIT method for two GID and LID sources with central DOAs $10^{\circ}$ and $30^{\circ}$ at $\mathrm{SNR}=10 \mathrm{~dB}$

central DOA estimates by the FAPI-TLS-ESPRIT algorithm, their values are close to those obtained with our proposed method. Furthermore, comparing Figs. 11 and 12 to Figs. 8 and 9 of the second example, we see clearly that the performances of the FAPI-TLS-ESPRIT method increase in particular at high SNR values. Therefore, we can conclude that the improvement made by our method with regard to the FAPI-TLS-ESPRIT method is more prominent for low SNR values.
Next, we reconsider the low SNR assumption (SNR = $0 \mathrm{~dB}$ ) but we consider two other ID sources. The first one is assumed to be uniform distributed (UID) with central DOA $\bar{\theta}_{1}=10^{\circ}$ and angular spread $\sigma_{1}=2.5^{\circ}$ and the second one GID with central DOA $\bar{\theta}_{2}=30^{\circ}$ and angular spread $\sigma_{2}=1.5^{\circ}$.

Figures 13,14, and 15 exhibit the tracking performances of our proposed algorithm and the FAPI-TLS-ESPRIT algorithm.

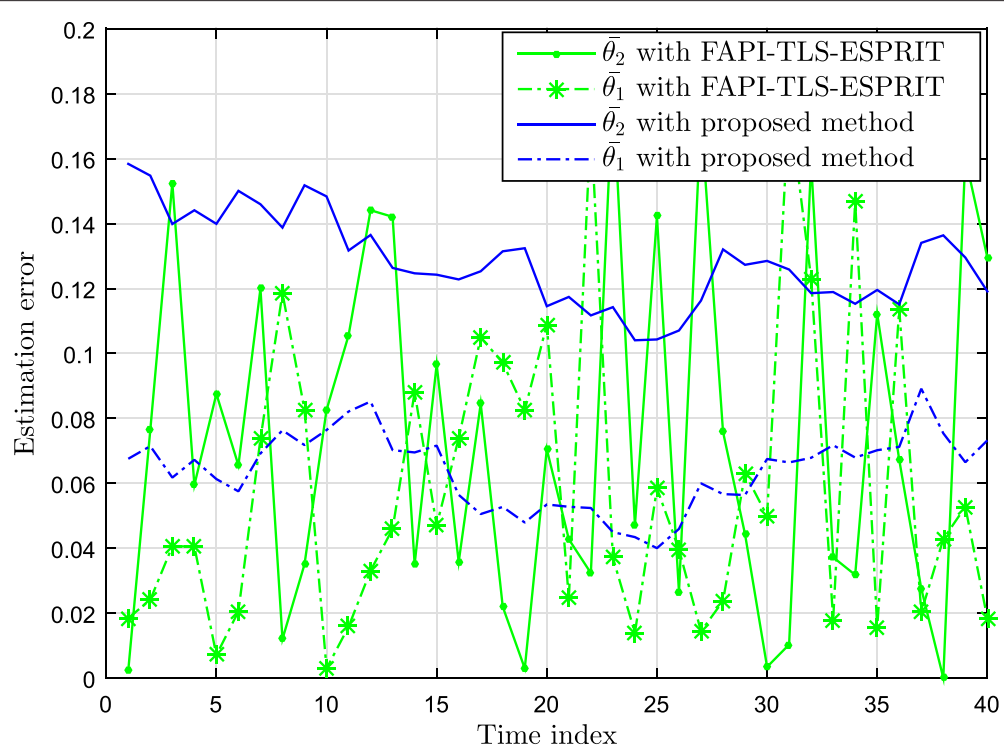

Fig. 11 Estimation errors made by the proposed algorithm and the FAPI-TLS-ESPRIT method for two GID and LID sources with central DOAs $10^{\circ}$ and $30^{\circ}$ at $\mathrm{SNR}=10 \mathrm{~dB}$ 


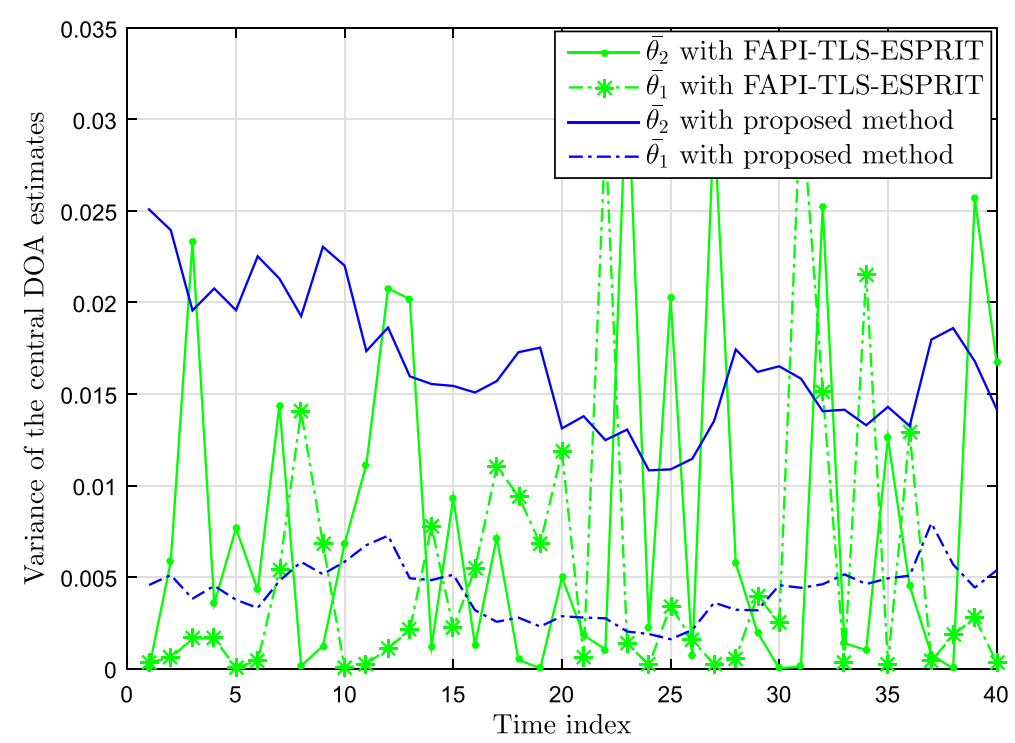

Fig. 12 Variances of the estimated central DOAs of the proposed algorithm and the FAPI-TLS-ESPRIT method for two GID and LID sources with central $\mathrm{DOAs} 10^{\circ}$ and $30^{\circ}$ at $\mathrm{SNR}=10 \mathrm{~dB}$

It can be seen from Fig. 13 that the tracking trajectory of the central DOA $\bar{\theta}_{1}$ of the UID source obtained with our proposed method deviates from the corresponding true trajectory. This is more illustrated by Figs. 14 and 15 where the corresponding estimation error is almost equal to 0.4 and the variance of $\widehat{\bar{\theta}}_{1}$ is near to 0.2 . Now, we compare Fig. 14 to Fig. 8 of the second example. These two figures are obtained in the same conditions but the only difference is that we assume UID and GID sources in Fig. 14 instead of GID and LID sources in Fig. 8. We notice here that the estimation error made by our proposed method is lower than 0.2 assuming Gaussian and Laplacian distributions. However, it exceeds 0.3 when the source is UID. Therefore, we can conclude that the proposed method can better track the central DOA when the source is GID or LID than when it is UID. This can be explained by the fact that our method (in particular, the covariance-fitting-based method) depends on the form of

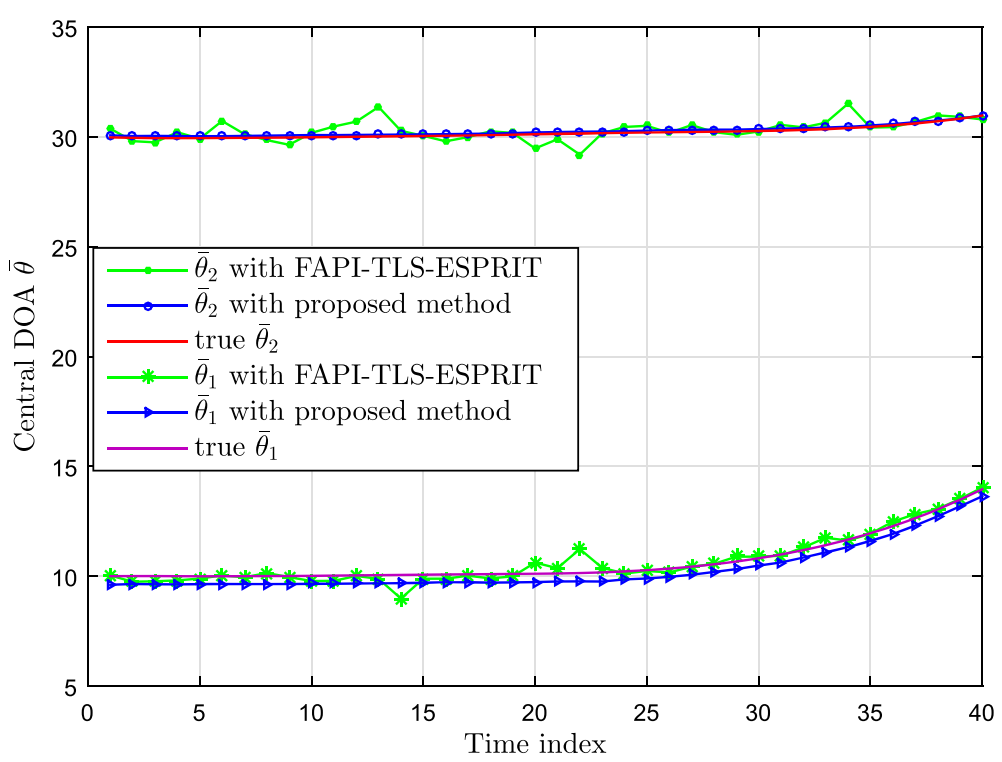

Fig. 13 Tracking trajectories using the proposed algorithm and the FAPI-TLS-ESPRIT method for two UID and GID sources with central DOAs $10^{\circ}$ and $30^{\circ}$ at $\mathrm{SNR}=0 \mathrm{~dB}$ 


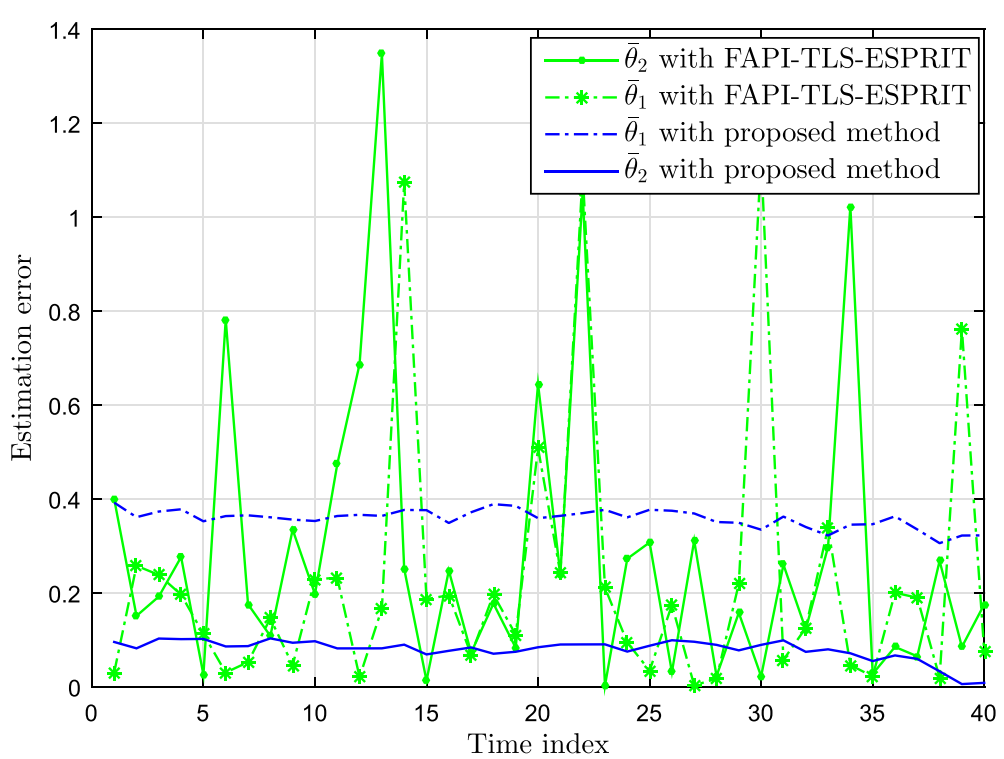

Fig. 14 Estimation errors made by the proposed algorithm and the FAPI-TLS-ESPRIT method for two UID and GID sources with central DOAs $10^{\circ}$ and $30^{\circ}$ at $\mathrm{SNR}=0 \mathrm{~dB}$

the angular distribution function in the estimation of the central DOA. However, we show from Figs. 13, 14, and 15 that regardless of the form of the angular distribution, our proposed algorithm outperforms the FAPI-TLS-ESPRIT method. Therefore, we can conclude that even if our new method depends on the form of the angular distribution, its performances remain satisfactory, whatever the considered form, by comparing it to the FAPI-TLS-ESPRIT method. Now, we reconsider in Figs. 16, 17, and 18 two
GID and LID sources. The first one is assumed to be GID with central DOA $\bar{\theta}_{1}=10^{\circ}$ and angular spread $\sigma_{1}=1^{\circ}$ and the second one is LID with central DOA $\bar{\theta}_{2}=17^{\circ}$ and angular spread $\sigma_{2}=2.5^{\circ}$.

It can be seen from these figures that our proposed method outperforms the FAPI-TLS-ESPRIT method even if the sources are closely spaced. However, comparing these figures to Figs. 2, 3, and 4 of the first example and Figs. 7, 8, and 9 of the second example where the sources

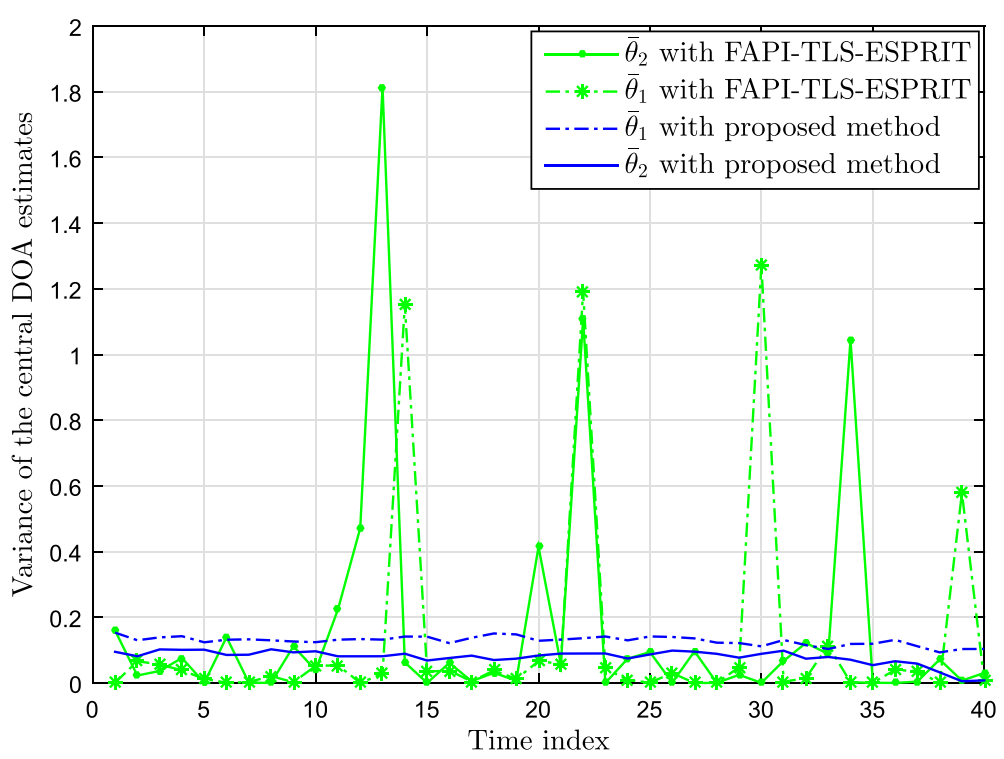

Fig. 15 Variances of the estimated central DOAs of the proposed algorithm and the FAPI-TLS-ESPRIT method for two UID and GID sources with central DOAs $10^{\circ}$ and $30^{\circ}$ at $\mathrm{SNR}=0 \mathrm{~dB}$ 


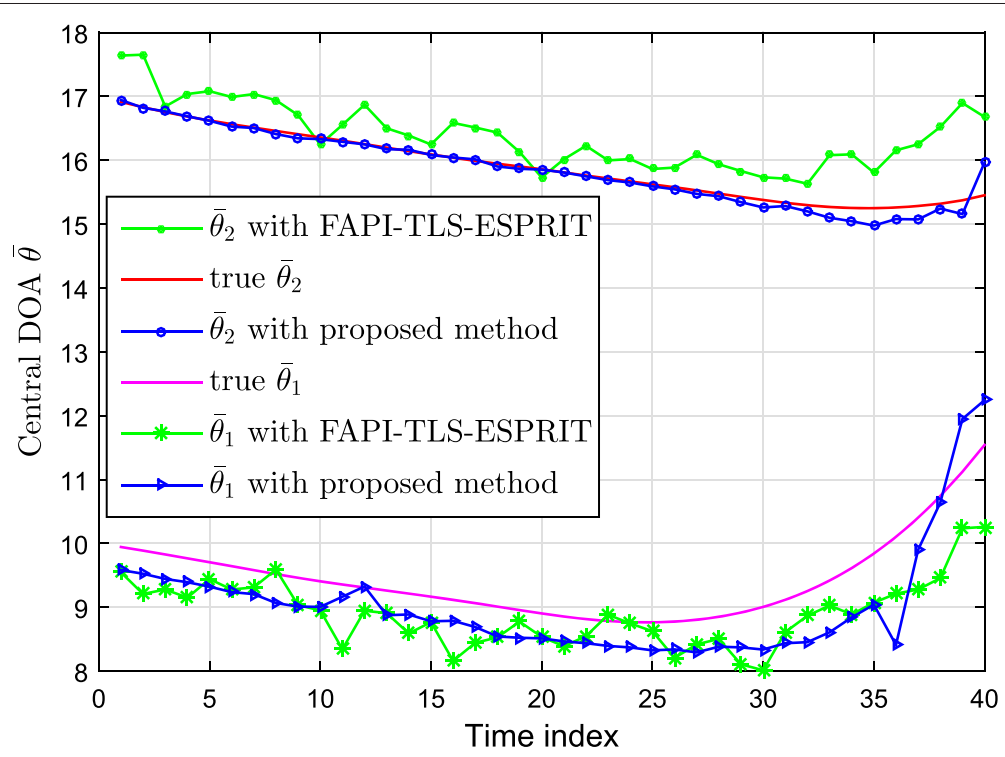

Fig. 16 Tracking trajectories using the proposed algorithm and the FAPI-TLS-ESPRIT method for two GID and LID sources with central DOAs $10^{\circ}$ and $17^{\circ}$ at $S N R=0 \mathrm{~dB}$

are widely spaced, we note that the proposed method cannot properly track the DOAs when the sources are closely spaced. This confirms the conclusion previously proved: the more the sources are spaced, the more the proposed method can exactly track the DOAs.

Finally, we consider in Figs. 19 and 20 three sources. The first one is assumed to be GID with central DOA $\bar{\theta}_{1}=10^{\circ}$ and angular spread $\sigma_{1}=1^{\circ}$, the second one is LID with central DOA $\bar{\theta}_{2}=20^{\circ}$ and angular spread $\sigma_{2}=1.5^{\circ}$, and the third one is GID with central DOA $\bar{\theta}_{2}=35^{\circ}$ and angular spread $\sigma_{2}=2.5^{\circ}$. Figure 19 presents the tracking trajectories of the central DOAs estimated by our proposed method, while Fig. 20 displays the tracking trajectories of the central DOAs estimated by the FAPI-TLS-ESPRIT method. We see clearly from these figures that our proposed method outperforms by far the FAPI-TLS-ESPRIT method when the number of sources increases. However, comparing Fig. 19 with other corresponding figures when

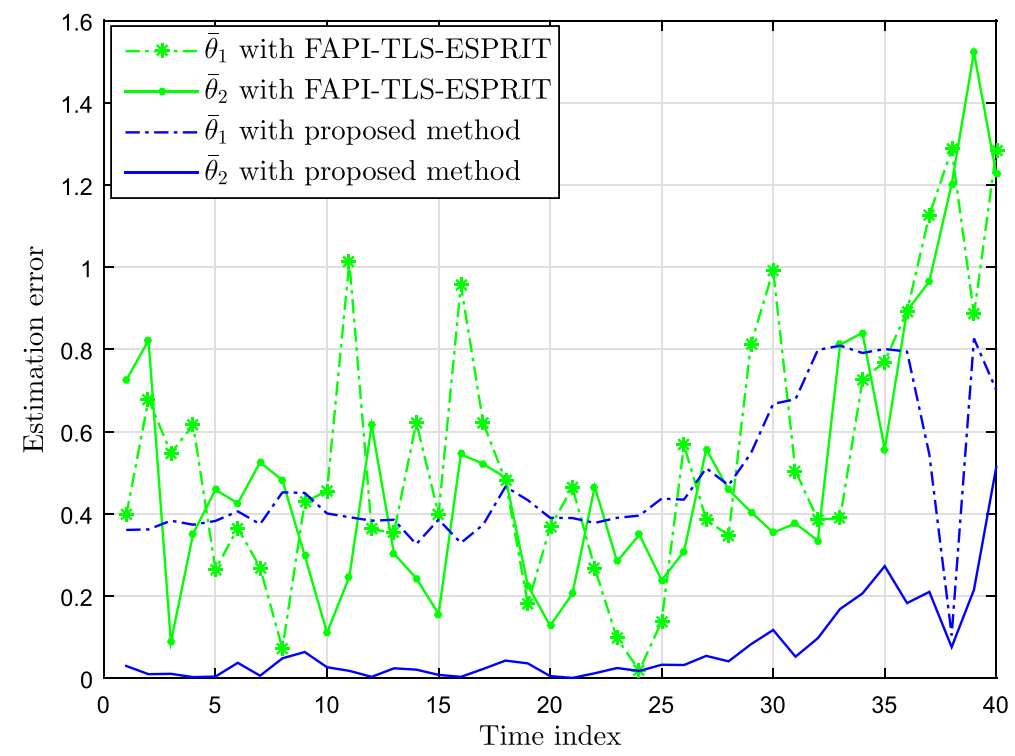

Fig. 17 Estimation errors made by the proposed algorithm and the FAPI-TLS-ESPRIT method for two GID and LID sources with central DOAs $10^{\circ}$ and $17^{\circ}$ at $S N R=0 \mathrm{~dB}$ 


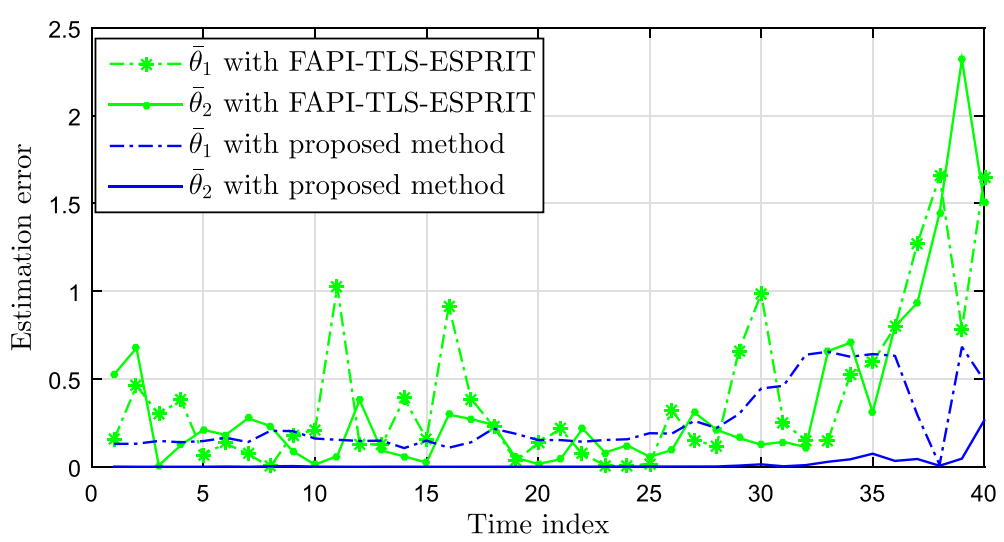

Fig. 18 Variances of the estimated central DOAs of the proposed algorithm and the FAPI-TLS-ESPRIT method for two GID and LID sources with central DOAs $10^{\circ}$ and $17^{\circ}$ at $\mathrm{SNR}=0 \mathrm{~dB}$

we only consider two ID sources, we show that the performances of the proposed method degrade especially for high time index. Therefore, we can conclude that although our new method outperforms the FAPI-TLS-ESPRIT, its performances degrade significantly when the number of sources increases and they are closely spaced.

\section{Conclusions}

In this paper, we developed a new method for tracking the central DOAs assuming multiple incoherently distributed (ID) sources. This method is based on a simple covariance fitting optimization technique to estimate the central DOAs in each observed time interval. It also uses the Kalman filter to model the mobility of the sources and track the different DOAs during the tracking period. Our method was compared to the FAPI-TLS-ESPRIT algorithm using the TLS-ESPRIT method and the subspace updating via FAPI-algorithm in different scenarios. We proved that this new method outperforms the FAPI-TLSESPRIT method. The improvement made by our method with regard to the FAPI-TLS-ESPRIT method is more prominent for low SNR values. We also showed that the proposed method can better track the central DOA when the source is GID or LID than when it is UID. Therefore, our method depends on the form of the angular distribution function when tracking the central DOAs. Moreover, we proved that the more the sources are spaced, the more the proposed method can exactly track the central DOAs. Finally, when the number of sources increases, the performances of our proposed algorithm decrease.

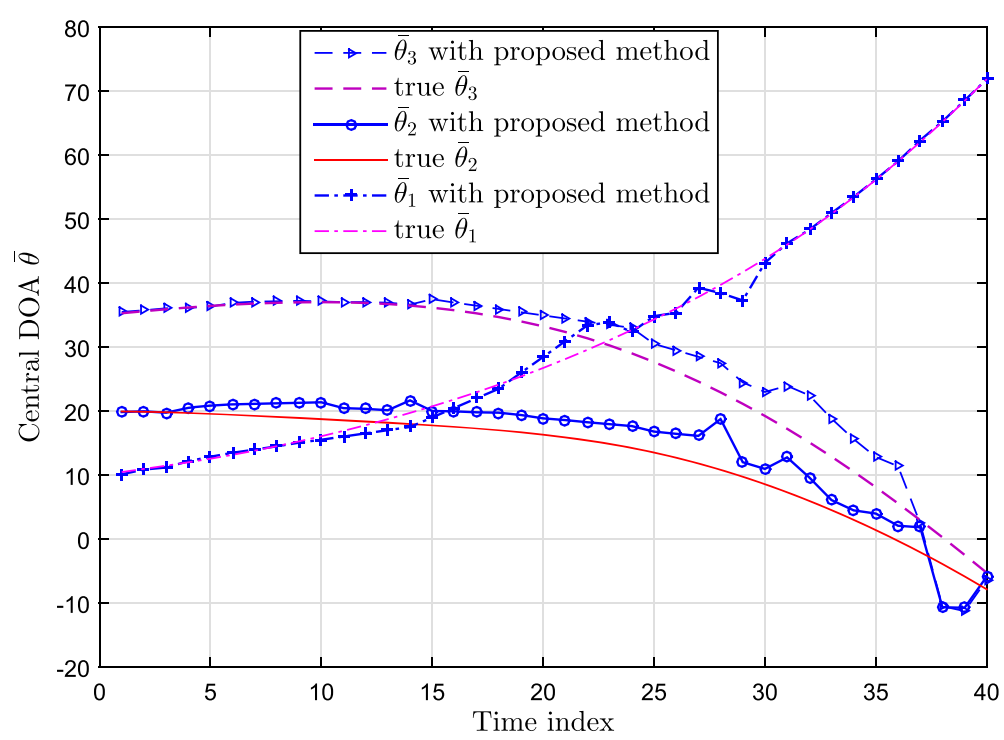

Fig. 19 Tracking trajectories using the proposed algorithm for three GID, LID, and GID sources with central DOAs $10^{\circ}, 20^{\circ}$, and $35^{\circ}$ at $S N R=0 \mathrm{~dB}$ 


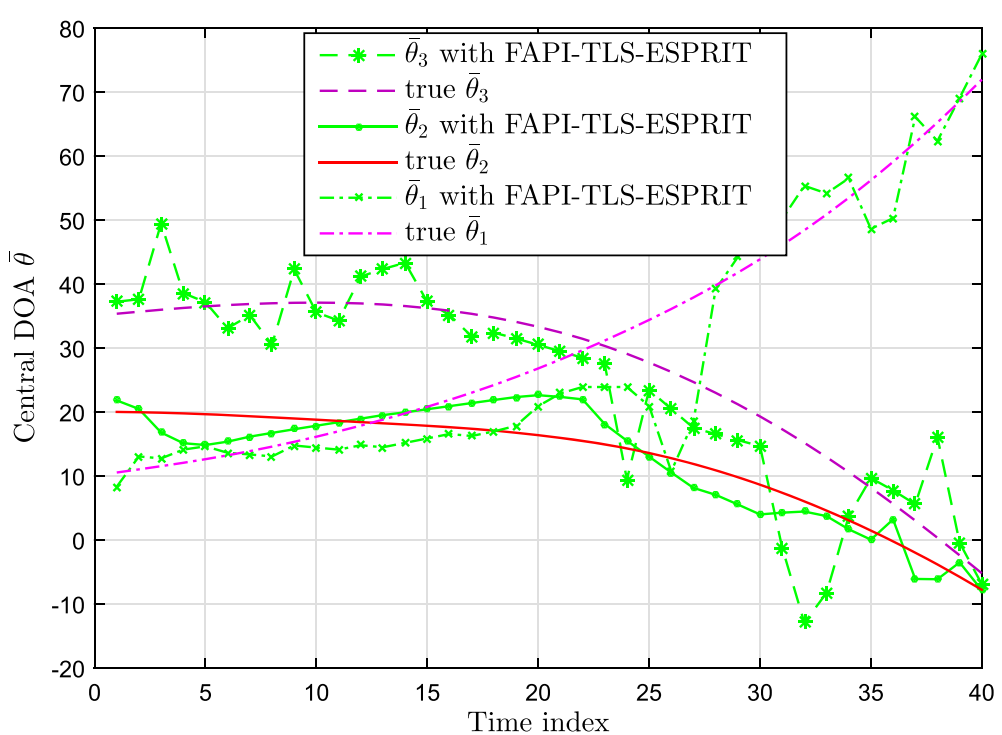

Fig. 20 Tracking trajectories using the FAPI-TLS-ESPRIT method for for three GID, LID, and GID sources with central DOAs $10^{\circ}, 20^{\circ}$, and $35^{\circ}$ at $\mathrm{SNR}=0 \mathrm{~dB}$

\section{Endnote}

${ }^{1}$ We assume that the delay spread caused by the multipath propagation is small compared to the inverse bandwidth of the transmitted signals. This means that the narrowband assumption is valid in the presence of scattering.

\section{Competing interests}

The authors declare that they have no competing interests.

\section{Author details}

${ }^{1}$ Tunisia Polytechnic School, B.P. 743-2078, La Marsa, Tunisia. ${ }^{2}$ Wireless Communications Group, Institut National de la Recherche Scientifique, Centre Energie, Matériaux, et Télécommunications, 800, de la Gauchetière Ouest, Bureau 6900, Montreal QC H5A 1 K6, Canada.

Received: 19 April 2015 Accepted: 15 October 2015

Published online: 02 November 2015

\section{References}

1. PStoica, A Nehorai, MUSIC, Maximum likelihood, and Cramér-Rao bound: further results and comparisons. IEEE Trans. Acoust., Speech, Sig. Process. 38, 2140-2150 (1990)

2. R Roy, T Kailath, AB Gershman, ESPRIT, estimation of signal parameters via rotational invariance techniques. IEEE Trans. Acoust. Speech Sig. Process. 37, 984-995 (1989)

3. F Haddadi, MM Nayebi, MR Aref, Direction-of-arrival estimation for temporally correlated narrowband signals. IEEE Trans. Sig. Process. 57, 600-609 (2009)

4. S Ben Hassen, F Bellili, A Samet, S Affes, DOA Estimation of temporally and spatially correlated narrowband noncircular sources in spatially correlated white noise. IEEE Trans. Sig. Process. 59, 4108-4121 (2011)

5. S Valaee, B Champagne, P Kabal, Parametric localization of distributed sources. IEEE Trans. Sig. Process. 43(9), 2144-2153 (1995)

6. R Raich, J Goldberg, H Messor, Bearing estimation for a distributed source: modeling, inherent accuracy limitations and algorithms. IEEE Trans. Sig. Process. 48(2), 429-441 (2000)

7. TTrump, B Ottersten, Estimation of nominal direction of arrival and angular spread using an array of sensors. Sig. Process. 50(1-2), 57-70 (1996)
8. O Besson, F Vincent, P Stoica, AB Gershman, Approximate maximum likelihood estimators for array processing in multiplicative noise environments. IEEE Trans. Sig. Process. 48, 2506-2518 (2000)

9. O Besson, P Stoica, A fast and robust algorithm for DOA estimation of a spatially dispersed source. Digital Signal Process. 9, 267-279 (1999)

10. O Besson, P Stoica, AB Gershman, A simple and accurate direction of arrival estimator in the case of imperfect spatial coherence. IEEE Trans. Signal Process. 49, 730-737 (2001)

11. O Besson, P Stoica, Decoupled estimation of DOA and angular spread for a spatially distributed source. IEEE Trans. Sig. Process. 48, 1872-1882 (2000)

12. A Zoubir, Y Wang, P Chargé, A modified COMET-EXIP method for estimating a scattered source. Elsevier Signal Process. J. 86, 733-743 (2006)

13. J Lee, J Joung, JD Kim, A method for the direction-of-arrival estimation of incoherently distributed sources. IEEE Trans. Vehicular Technol. 57(5), 2885-2893 (2008)

14. Y Meng, P Stoica, KM Wong, Estimation of the directions of arrival of spatially dispersed signals in array processing. Proc. Inst. Elect. Eng.-Radar, Sonar Navigat. 143, 1-9 (1996)

15. M Bengtsson, Antenna array signal processing for high rank models (1999). Ph.D. dissertation, Signals, Sensors, Syst. Dept., Royal Institute of Technology, Stockholm, Sweden

16. Sh Shahbazpanahi, Sh Valaee, MH Bastani, Distributed source localization using ESPRIT algorithm. IEEE Trans. Sig. Process. 49(10), 2169-2178 (2001)

17. S Shahbazpanahi, AB Gershman, Z-Q Luo, KM Wong, Robust adaptive beamforming for general-rank signal models. IEEE Trans. Sig. Process. 51(9), 2257-2269 (2003)

18. A Hassanien, S Shahbazpanahi, AB Gershman, A generalized Capon estimator for localization of multiple spread sources. IEEE Trans. Sig. Process. 52(1), 280-283 (2004)

19. Sh Shahbazpanahi, Sh Valaee, AB Gershman, A covariance fitting approach to parametric localization of multiple incoherently distributed sources. IEEE Trans. Sig. Process. 52(3), 592-600 (2004)

20. A Zoubir, Y Wang, P Chargé, Efficient subspace-based estimator for localization of multiple incoherently distributed sources. IEEE Trans. Sig. Process. 56(2), 532-542 (2008)

21. B Yang, Projection approximation subspace tracking. IEEE Trans. Sig. Process. 44(1), 95-107 (1995)

22. K Abed-Meraim, A Chkeif, Y Hua, Fast orthonormal PAST algorithm. IEEE Sig. Proc. Lett. 7(3), 60-62 (2000)

23. J Sanchez-Araujo, S Marcos, An Efficient PASTd-Algorithm implementation for multiple direction of arrival tracking. IEEE Trans. Sig. Process. 47(8), 2321-2324 (1999) 
24. R Badeau, B David, G Richard, Fast approximated power iterations for fast subspace tracking. IEEE Trans. Sig. Process. 53(8), 2931-2941 (2005)

25. Sh-Y Hou, H-S Hung, Sh-H Chang, J-Ch Liu, Novel algorithms for tracking multiple targets. J. Marine Sci. Technol. 18(2), 259-267 (2010)

26. B Liao, ZG Zhang, SC Chan, DOA Estimation and tracking of ULAs with mutual coupling. IEEE Trans. Aero. Elect. Syst. 48(1), 891-905 (2012)

27. JF Gu, SC Chan, WP Zhu, MNS Swamy, Sparse linear arrays for estimating and tracking DOAs of signals with known waveforms. IEEE Trans. Circ. Syst. 60(1), 46-50 (2013)

28. G Xiansheng, W Qun, H Anmin, B Danping, Fast DOA tracking of multiple scattering sources based on subspace updating. Proc. Comm. Circ. and Syst., 713-717 (2007)

29. Y Han, J Wang, Q Zhao, J Gao, An efficient DOA tracking algorithm for coherently distributed source. IEEE Conf. Commun. Inf. Techn, 493-496 (2011)

30. P Zetterberg, Mobile cellular communications with base station antenna arrays: spectrum efficiency, algorithms, and propagation models (1997). Ph.D. dissertation, Royal Inst. Technol., Stockholm, Sweden

31. Y Bar-Shalom, TE Fortmann, Tracking and Data Association. (Academic, New York, 1988)

32. RJ Serfling, Approximation Theorems of Mathematical Statistics. (Wiley, New York, 1980)

33. J-P Delmas, Y Meurisse, Asymptotic performance analysis of DOA finding algorithms with temporally correlated narrowband signals. IEEE Trans. Sig. Process. 48(9), 2669-2674 (2000)

34. CR Rao, CR Sastry, B Zhou, Tracking the direction of arrival of multiple moving targets. IEEE Trans. Sig. Process. 42(5), 1133-1144 (1994)

35. M Ghogho, O Besson, A Swami, Estimation of directions of arrival of multiple scattered sources. IEEE Trans. Sig. Process. 49(11), 2467-2480 (2001)

36. P Stoica, R Moses, Introduction to Spectral Analysis. (Prentice-Hall, Upper Saddle River, NJ, 1997)

\section{Submit your manuscript to a SpringerOpen ${ }^{\circ}$ journal and benefit from:}

- Convenient online submission

- Rigorous peer review

- Immediate publication on acceptance

- Open access: articles freely available online

- High visibility within the field

- Retaining the copyright to your article

Submit your next manuscript at $\boldsymbol{\nabla}$ springeropen.com 